\title{
JACOBSON-MOROZOV LEMMA FOR ALGEBRAIC SUPERGROUPS
}

\author{
INNA ENTOVA-AIZENBUD, VERA SERGANOVA
}

\begin{abstract}
Given a quasi-reductive algebraic supergroup $G$, we use the theory of semisimplifications of symmetric monoidal categories to define a symmetric monoidal functor $\Phi_{x}: \operatorname{Rep}(G) \rightarrow \operatorname{Rep}(O S p(1 \mid 2))$ associated to any given element $x \in \operatorname{Lie}(G)_{\overline{1}}$. For nilpotent elements $x$, we show that the functor $\Phi_{x}$ can be defined using the Deligne filtration associated to $x$.

We use this approach to prove an analogue of the Jacobson-Morozov Lemma for algebraic supergroups. Namely, we give a necessary and sufficient condition on odd nilpotent elements $x \in \operatorname{Lie}(G)_{\overline{1}}$ which define an embedding of supergroups $O S p(1 \mid 2) \rightarrow G$ so that $x$ lies in the image of the corresponding Lie algebra homomorphism.
\end{abstract}

\section{InTRODUCTION}

1.1. Let $\mathbb{k}$ be an algebraically closed field of characteristic zero.

In the classical setting, one has the following version of the Jacobson-Morozov lemma: given an embedding of algebraic groups $\mathbb{G}_{a} \rightarrow L$, where $L$ is reductive and $\mathbb{G}_{a}$ is the additive group, one can extend this homomorphism to a homomorphism $S L_{2} \rightarrow L$.

The latter homomorphism is unique up to conjugation by an element of $L$.

The embedding $\mathbb{G}_{a} \rightarrow L$ of course corresponds to a choice of nilpotent element in Lie $(L)$. This provides the Jacobson-Morozov lemma for Lie algebras: every nilpotent element in a semisimple Lie algebra $\mathfrak{l}$ can be embedded into an $\mathfrak{s l}_{2}$-subalgebra of $\mathfrak{g}$, and this embedding is unique up to conjugation by an element of $L$.

In this paper, we extend this result to the case of a quasi-reductive algebraic supergroup $G$ (here "quasi-reductive" means that the even part $G_{\overline{0}}$ of the supergroup $G$ is a reductive algebraic group).

The additive group $\mathbb{G}_{a}$ will then be replaced by a supergroup $\mathbb{G}_{a}^{(1 \mid 1)}$ whose Lie superalgebra $\mathfrak{g}_{a}^{(1 \mid 1)}$ is a nilpotent Lie superalgebra, spanned by an odd nilpotent element $x$ and its commutator $[x, x]$.

Fix an algebraic supergroup $G$ with corresponding Lie superalgebra $\mathfrak{g}=\operatorname{Lie}(G)$ and an odd nilpotent element $y \in \mathfrak{g}_{\overline{1}}$. We then have an homomorphism $\mathbb{G}_{a}^{(1 \mid 1)} \rightarrow G$ of algebraic supergroups, whose differential $\mathfrak{g}_{a}^{(1 \mid 1)} \rightarrow \mathfrak{g}_{\overline{1}}$ sends $x$ to $y$.

The group $S L_{2}$ will be replaced by the supergroup $O S p(1 \mid 2)$, the latter being one of a few algebraic supergroups whose category of finite-dimensional representations is semisimple. The group $\mathbb{G}_{a}^{(1 \mid 1)}$ embeds into $O S p(1 \mid 2)$, and $\mathfrak{g}_{a}^{(1 \mid 1)}$ is isomorphic to a maximal nilpotent subalgebra of $\mathfrak{o s p}(1 \mid 2)$.

One should state right away that the situation here is trickier than in the classical setting: we do not expect an embedding $\mathbb{G}_{a}^{(1 \mid 1)} \rightarrow G$ (corresponding to a choice of an odd nilpotent element in $\mathfrak{g})$ to necessarily give an embedding $O S p(1 \mid 2) \rightarrow G$. Indeed, the irreducible finite-dimensional representations of $O S p(1 \mid 2)$ have categorical dimension (also called "superdimension") \pm 1 , therefore we must have that the restriction of any 
finite-dimensional $G$-module to $\mathbb{G}_{a}^{(1 \mid 1)}$ is a direct sum of indecomposable $\mathbb{G}_{a}^{(1 \mid 1)}$-modules of categorical dimension \pm 1 .

To state our main result, we will use the following definition:

Definition. Let $V$ be a finite-dimensional vector superspace with an action of $\mathbb{G}_{a}^{(1 \mid 1)}$ on it.

The action is called neat if as a $\mathbb{G}_{a}^{(1 \mid 1)}$-module, all the indecomposable summands of $V$ have non-zero categorical dimension ("superdimension").

The action of $\mathbb{G}_{a}^{(1 \mid 1)}$ on $V$ is completely determined by the odd nilpotent operator $x \in \operatorname{End}(V)$. The operator $x$ is called neat if it defines a neat action of $\mathbb{G}_{a}^{(1 \mid 1)}$ on $V$.

Our main result is the following "odd" version of Jacobson-Morozov Lemma in the superalgebra setting (see Theorem 4.2.1):

Theorem 1. Let $G$ be a quasi-reductive algebraic supergroup, and $\mathfrak{g}=\operatorname{Lie}(G)$ its Lie superalgebra. Let $x \in \mathfrak{g}_{\overline{1}}, x \neq 0$ be a nilpotent element such that $\left.x\right|_{V}$ is neat, for any finite dimensional (algebraic) representation $V$ of $G$.

Let $i: \mathbb{G}_{a}^{(1 \mid 1)} \hookrightarrow G$ be the homomorphism of algebraic supergroups corresponding to the inclusion $x \in \mathfrak{g}$.

Then the inclusion $i$ can be extended to an injective homomorphism $\bar{i}: O S p(1 \mid 2) \hookrightarrow G$.

Moreover, we give a simple criterion to check that an element $x \in \mathfrak{g}_{\overline{1}}$ is neat: given a quasi-reductive algebraic supergroup $G$, a faithful representation $V$ of $G$, and $x \in \mathfrak{g}_{\overline{1}} \backslash\{0\}$, we prove that the operator $\left.x\right|_{V}$ is nilpotent and neat iff $x$ satisfies the conditions of Theorem 1.

Remark 2. As it was stated above, the neatness of $x$ is also a necessary condition in order for the homomorphism $\bar{i}$ extending $i$ to exist.

Let us give a short overview of our main tool for proving Theorem 1 .

Consider the category $\operatorname{Rep}\left(\mathbb{G}_{a}^{(1 \mid 1)}\right)$. Using the theory of semisimplification, we show that there exists a (non-exact) $\mathbb{k}$-linear full symmetric monoidal functor

$$
S: \operatorname{Rep}\left(\mathbb{G}_{a}^{(1 \mid 1)}\right) \rightarrow \operatorname{Rep}(O S p(1 \mid 2))
$$

making $\operatorname{Rep}(O S p(1 \mid 2))$ the universal semisimple quotient of the category $\operatorname{Rep}\left(\mathbb{G}_{a}^{(1 \mid 1)}\right)$. The functor $S$ annihilates all indecomposable $\mathbb{G}_{a}^{(1 \mid 1)}$-representations of superdimension zero.

Now, let us go back to the setting of Theorem 11. Consider the restriction functor $R_{x}: \operatorname{Rep}(G) \rightarrow \operatorname{Rep}\left(\mathbb{G}_{a}^{(1 \mid 1)}\right)$. Setting

$$
\Phi_{x}:=S \circ R_{x}: \operatorname{Rep}(G) \longrightarrow \operatorname{Rep}(O S p(1 \mid 2)),
$$

we show that this functor is an exact symmetric monoidal functor between superTannakian categories. Therefore it defines a homomorphism $\bar{i}$ as in Theorem 1 .

Another construction of $\Phi_{x}$ is as follows. Let $M \in \operatorname{Rep}(G)$, and consider the action of $x$ on it. This action defines a canonical finite increasing "Deligne" filtration

$$
\ldots \subset \mathcal{F}^{i}(M) \subset \mathcal{F}^{i+1}(M) \subset \ldots
$$

satisfying conditions similar to that of the classical Deligne filtration appearing in the Hodge theory.

Let $G r^{i}(M)=\mathcal{F}^{i}(M) / \mathcal{F}^{i-1}(M)$. Since $x$ is neat, we have: $G r^{2 i+1}(M)=0$ for all $i$. Then the grading and the action of $x$ extends uniquely to an action of $O S p(1 \mid 2)$ on $\bigoplus_{i} G r^{2 i}(M)$ and an isomorphism of $O S p(1 \mid 2)$-modules $\bigoplus_{i} G r^{2 i}(M) \cong \Phi_{x}(M)$. 
The nilpotent elements in $\mathfrak{g}_{\overline{1}}$ satisfying the condition on Theorem 1 are called neat elements, and the set of such elements is denoted $\mathfrak{g}_{\text {neat }}$.

We initiate the study of the set $\mathfrak{g}_{\text {neat }}$; it is stable under the adjoint action of $G_{\overline{0}}$ and if $G$ is quasi-reductive, we show that $\mathfrak{g}_{\text {neat }}$ has finitely many $G_{\overline{0}}$-orbits, and $\mathfrak{g}_{\overline{1}}=\mathfrak{g}_{\text {neat }}$ iff $\operatorname{Rep}(G)$ is semisimple.

The construction of the functor $\Phi_{x}$ using semisimplification is inspired by [EtO18], and can be extended to arbitrary odd elements in the Lie superalgebra $\mathfrak{g}$. Each element $x \in \mathfrak{g}_{\overline{1}}$ defines a functor $\Phi_{x}: \operatorname{Rep}(G) \longrightarrow \operatorname{Rep}(O S p(1 \mid 2))$ in a similar manner.

This allows one to define the notion of support of a module $M \in \operatorname{Rep}(G)$ as the subset

$$
\operatorname{supp}(M):=\left\{x \in \mathfrak{g}_{1} \mid \Phi_{x}(M) \neq 0\right\} .
$$

We study supports of modules in Section 6. We show that the minimal support a module can have is $\mathfrak{g}_{\text {neat }}$, and this occurs when $M$ is projective.

1.2. Acknowledgement. We would like to thank Joseph Bernstein, Pavel Etingof and Victor Ostrik for helpful discussions. The authors were supported by the NSF-BSF grant NSFMath 2019694.

\section{Notation}

Our base field will be an algebraically closed field $\mathbb{k}$ with $\operatorname{char}(\mathbb{k})=0$.

2.1. Tensor categories and vector superspaces. All our categories will be $\mathbb{k}$-linear rigid symmetric monoidal, with the bifunctor $-\otimes-$ being bilinear. All the functors will be symmetric monoidal and $\mathbb{k}$-linear.

We will write SM for short when refering to symmetric monoidal (both categories and functors).

Throughout the paper, we will use the following terminology and assumptions, following EtGNO15:

- A tensor category is an abelian rigid SM $\mathbb{k}$-linear category, where $-\otimes-$ is biexact 1 . A tensor functor between tensor categories is an exact SM functor.

- We will assume that $\operatorname{End}(\mathbb{1})=\mathbb{k}$ in all our categories.

- In a rigid $\mathrm{SM}$ category $\mathcal{U}$, one defines the trace of $f \in \operatorname{End}(C), C \in \mathcal{U}$ as $\operatorname{tr}(f) \in$ $\operatorname{End}(\mathbb{1})$ where

$$
\operatorname{tr}(F): \mathbb{1} \rightarrow C \otimes C^{*} \stackrel{f \otimes \operatorname{Id}_{C^{*}}}{\longrightarrow} C \otimes C^{*} \stackrel{b_{C, C^{*}}}{\longrightarrow} C^{*} \otimes C \rightarrow \mathbb{1} .
$$

The (categorical) dimension of an object $C$ is then defined as $\operatorname{dim}(C)=\operatorname{tr}\left(\operatorname{Id}_{C}\right) \in$ $\operatorname{End}(\mathbb{1})$.

A morphism $f: C_{1} \rightarrow C_{2}$ in $\mathcal{U}$ is called negligible if it satisfies the following condition:

$$
\forall g: C_{2} \rightarrow C_{1}, \operatorname{tr}(g \circ f)=0 .
$$

The set $\mathcal{N}$ of all negligible morphisms in a rigid SM $\mathbb{k}$-linear $\mathcal{U}$ forms an ideal under composition and tensor product, hence $\mathcal{U} / \mathcal{N}$ is again a rigid SM $\mathbb{k}$-linear category.

- The semisimplification of a rigid SM $\mathbb{k}$-linear category $\mathcal{U}$ is the pair $(S, \overline{\mathcal{U}})$, where $\overline{\mathcal{U}}=\mathcal{U} / \mathcal{N}$, and $S: \mathcal{U} \rightarrow \overline{\mathcal{U}}$ is the quotient functor.

One can immediately see that $\overline{\mathcal{U}}$ is a semisimple rigid SM $\mathbb{k}_{k}$-linear category, and $S: \mathcal{U} \rightarrow \overline{\mathcal{U}}$ is a full SM $\mathbb{k}$-linear functor. In fact, the pair $(S, \overline{\mathcal{U}})$ is universal among pairs, cf. for example [AK02, EtO18], and also [H15, BEEO20].

\footnotetext{
${ }^{1}$ In fact, this follows from bilinearity of $-\otimes-$.
} 
Two important examples of rigid SM categories are the category of finite-dimensional representations of a group $G$ and the category of finite-dimensional vector superspaces sVect, defined below.

2.2. Vector superspaces and supergroups. A vector superspace is a $\mathbb{Z} / 2 \mathbb{Z}$-graded $\mathbb{K}^{-}$ vector space $V=V_{\overline{0}} \oplus V_{\overline{1}}$; for $v \in V_{\varepsilon}, \varepsilon \in \mathbb{Z} / 2 \mathbb{Z}$, we denote by $\bar{v}=\varepsilon$ the parity of $v$. Given two superspaces $V, W$, the space $\operatorname{Hom}_{\mathbb{k}}(V, W)$ is naturally $\mathbb{Z} / 2 \mathbb{Z}$-graded as well, with homogeneous morphisms called even and odd respectively.

The objects in the category of vector superspaces sVect are finite-dimensional vector superspaces and the morphisms are linear even morphisms:

$$
\operatorname{Hom}_{\text {svect }}(V, W)=\operatorname{Hom}_{\mathbb{k}}(V, W)_{\overline{0}} .
$$

The category sVect has a monoidal structure given by $\left(\otimes, \mathbb{k}^{1 \mid 0}\right)$, with the symmetry morphisms

$$
b_{V, W}: V \otimes W \rightarrow W \otimes V, \quad v \otimes w \mapsto(-1)^{\bar{v}} \bar{w} w \otimes v
$$

This makes sVect a rigid SM category, which is not equivalent to the SM category $\operatorname{Rep}(\mathbb{Z} / 2 \mathbb{Z})$.

The (categorical) dimension of $V \in$ sVect, also called superdimension, is then $\operatorname{dim} V=$ $\operatorname{dim} V_{\overline{0}}-\operatorname{dim} V_{\overline{1}}$. Sometimes we also denote the dimension of $V$ as a vector space by $\left(\operatorname{dim} V_{\overline{0}} \mid \operatorname{dim} V_{\overline{1}}\right)$.

We will denote by $\Pi$ the change of parity endofunctor on sVect: namely, $\Pi \mathbb{k}^{m \mid n} \cong \mathbb{k}^{n \mid m}$.

In the category sVect one can define Lie algebra objects, called (finite-dimensional) Lie superalgebras. An example of such an object is the vector superspace $\operatorname{End}(V)$ with a (signed) commutator bracket, denoted $\mathfrak{g l}(V)$. For the vector superspace $V=\mathbb{k}^{m \mid n}$, we denote $\mathfrak{g l}(m \mid n)=\mathfrak{g l}(V)$.

Similarly, one can consider algebraic (affine) supergroup 2]: these form a category which is opposite to the category of finitely-generated commutative Hopf algebra ind-objects in sVect, and each algebraic supergroup $G$ has a (finite-dimensional) Lie superalgebra $\operatorname{Lie}(G)$ attached to it.

A pro-supergroup $G$ is a limit of supergroups; its algebra of functions $O(G)$ is a Hopf superalgebra, not necessarily finitely generated.

Definition 2.2.1. Given a Lie superalgebra $\mathfrak{g}$, the category of its (finite-dimensional) representations $\operatorname{Rep}(\mathfrak{g})$ has objects $(V, \rho)$ where $V \in \operatorname{sVect}$ and $\rho: \mathfrak{g} \rightarrow \mathfrak{g l}(V)$ is a homomorphism of Lie algebra objects in sVect. The maps in $\operatorname{Rep}(\mathfrak{g})$ would be $\mathfrak{g}$-equivariant (even) maps of vector superspaces.

Definition 2.2.2. Given a supergroup $G$, we define $\operatorname{Rep}(G)$ as the category of representations of $G$ in sVect.

Let $G$ be an algebraic supergroup with Lie superalgebra $\mathfrak{g}$ and with the underlying (affine) algebraic group $G_{\overline{0}}$. A $\left(\mathfrak{g}, G_{\overline{0}}\right)$-module is by definition a $\mathfrak{g}$-module and $G_{\overline{0}}$-module such that the differential of the $G_{\overline{0}}$-action on $M$ coincides with the $\mathfrak{g}_{\overline{0}}$-action.

Theorem 2.2.3. Mas11] The category $\operatorname{Rep}(G)$ is equivalent to the category of finitedimensional $\left(G, \mathfrak{g}_{\overline{0}}\right)$-modules.

Remark 2.2.4. Any algebraic (affine) supergroup has a faithful finite-dimensional representation. This is proved in the same way as for affine algebraic groups (see e.g. Mil17, Chapter 4, Par. 9]).

\footnotetext{
${ }^{2}$ By "algebraic (super)group" we always mean an affine algebraic group (super)scheme $G$; its Hopf (super)algebra $O(G)$ is finitely generated.
} 
Definition 2.2.5. A supergroup $G$ is called quasi-reductive if $G_{\overline{0}}$ is reductive 3 .

2.3. Tannakian categories. In this section we use the terminology of [De02].

For a pre-Tannakian category $\mathcal{T}$ and a $\mathcal{T}$-group $G$, we have a group homomorphism $\varepsilon: \pi(\mathcal{T}) \rightarrow G$, where $\pi(\mathcal{T})$ denotes the fundamental group of $\mathcal{T}$, in the sense of [De02]. Define $\operatorname{Rep}_{\mathcal{T}}(G, \varepsilon)$ to be the category of representations $M$ of $G$ in $\mathcal{T}$ whose composition with $\varepsilon$ is the natural $\pi(\mathcal{T})$-action on $M$, seen as an object in $\mathcal{T}$.

If $\mathcal{T}=\operatorname{Rep}\left(G^{\prime}\right)$, we write $\operatorname{Rep}_{G^{\prime}}(G, \varepsilon)=\operatorname{Rep}_{\mathcal{T}}(G, \varepsilon)$ for short.

Deligne's theorem on super-Tannakian reconstruction states that given a finitely $\otimes$ generated pre-Tannakian category $\mathcal{T}$ where each object is annihilated by some Schur functor, the category $\mathcal{T}$ is equivalent to $\operatorname{Rep}_{\text {svect }}(G, \varepsilon)$ for some algebraic supergroup $G$ and $\varepsilon: \mu_{2} \rightarrow G$, where $\mu_{2} \cong \pi$ (sVect $) \cong\{ \pm 1\}$ and $\varepsilon$ is the corresponding supergroup homomorphism.

We now prove give a " categorical characterization of quasi-reductive supergroups".

Proposition 2.3.1. Let $G$ be an algebraic supergroup. Then $\operatorname{Rep}(G)$ has enough projectives if and only if $G$ is quasi-reductive.

Remark 2.3.2. Clearly, the existence of enough projectives in $\operatorname{Rep}(G, \varepsilon)$ is equivalent to the existence of enough projectives in $\operatorname{Rep}(G)$.

Proof. The induction $\operatorname{Ind}_{G_{\overline{0}}}^{G}$ and restriction $\operatorname{Res}_{G_{0}}$ define two adjoint functors between $\operatorname{Rep}(G)$ and $\operatorname{Rep}\left(G_{\overline{0}}\right)$. In fact, taking into account Theorem 2.2 .3 , we have the natural isomorphism

$$
\operatorname{Ind}_{G_{\overline{0}}}^{G}(?) \cong \operatorname{Hom}_{U\left(\mathfrak{g}_{\overline{0}}\right)}(U(\mathfrak{g}), ?),
$$

which explains why in this case the induction is an exact functor; it maps a finitedimensional module to a finite-dimensional module, and every finite-dimensional module is a submodule of $\operatorname{Ind}_{G_{\overline{0}}}^{G} M$ for some $M \in \operatorname{Rep}\left(G_{\overline{0}}\right)$. By definition of the induction functor, if $M$ is an injective $G_{\overline{0}}$-module then $\operatorname{Ind}_{G_{\overline{0}}}^{G} M$ is an injective $G$-module. The existence of enough projectives is equivalent to existence of enough injectives by duality. Hence if $G_{\overline{0}}$ is reductive, the category $\operatorname{Rep}(G)$ has enough projectives.

For converse, consider the functor $J: \operatorname{Rep}\left(G_{\overline{0}}\right) \rightarrow \operatorname{Rep}(G)$ defined by

$$
J(M)=U(\mathfrak{g}) \otimes_{U\left(\mathfrak{g}_{\overline{0}}\right)} M .
$$

This functor is left-adjoint to $\operatorname{Res}_{G_{0}}$ and isomorphic to $\operatorname{Ind}_{G_{\overline{0}}}^{G}$ after some twist. We have

$$
\operatorname{Hom}_{G_{\overline{0}}}\left(X, \operatorname{Res}_{G_{\overline{0}}} Y\right) \cong \operatorname{Hom}_{G}(J(X), Y),
$$

which implies that $\operatorname{Res}_{G_{\overline{0}}}$ maps projectives to projectives and hence injectives to injectives. Therefore, if $\operatorname{Rep}(G)$ has enough projectives, the same is true for $\operatorname{Rep}\left(G_{\overline{0}}\right)$. If $P \in \operatorname{Rep}\left(G_{\overline{0}}\right)$ is projective, then $P \otimes P^{*}$ is projective. If $P \neq 0$ then $\operatorname{dim} P \neq 0$, and the trivial module $\mathbb{k}$ splits as a direct summand in $P \otimes P^{*}$. Therefore, $\mathbb{k}$ is projective. That means $\operatorname{Ext}_{G_{\overline{0}}}^{1}(\mathbb{k}, M)=0$ for any $M \in \operatorname{Rep}\left(G_{\overline{0}}\right)$ and therefore $\operatorname{Ext}_{G_{\overline{0}}}^{1}\left(\mathbb{k}, N^{*} \otimes M\right)=\operatorname{Ext}_{G_{\overline{0}}}^{1}(N, M)=$ 0 for any $N, M \in \operatorname{Rep}\left(G_{\overline{0}}\right)$. Therefore $G_{\overline{0}}$ is reductive.

2.4. The Duflo-Serganova functor $D S$. Let $\mathfrak{g}$ be a Lie superalgebra, and let $x \in \mathfrak{g}_{\overline{1}}$ be an odd element satisfying $[x, x]=0$. In DuS05] M. Duflo and V. Serganova defined a functor

$$
D S_{x}: \operatorname{Rep}(\mathfrak{g}) \longrightarrow \operatorname{Rep}\left(\mathfrak{g}_{x}\right), \quad M \longmapsto M_{x}:=\left.\operatorname{Ker} x\right|_{M} /\left.\operatorname{Im} x\right|_{M}
$$

where $\mathfrak{g}_{x}:=\operatorname{Ker}_{x} / \operatorname{Im~ad}_{x}$ is again a Lie superalgebra.

\footnotetext{
${ }^{3}$ By a reductive algebraic group we mean an algebraic group whose finite-dimensional (rational) representations form a semisimple category.
} 
Example 2.4.1. For $\mathfrak{g}=\mathfrak{g l}(m \mid n)$ and $x \in \mathfrak{g}_{\overline{1}}$ of rank 1, we have: $\mathfrak{g}_{x} \cong \mathfrak{g l}(m-1 \mid n-1)$.

This functor is symmetric monoidal (hence preserves categorical dimensions), but in general not exact on either side.

2.5. Representations of $O S p(1 \mid 2)$. Recall that all isomorphism classes of indecomposable representations of the usual additive algebraic group $\mathbb{G}_{a}$ are enumerated by their dimensions.

Let $V_{k}$ be the indecomposable $(k+1)$-dimensional representation of the usual additive algebraic group $\mathbb{G}_{a}$ (we set $V_{-1}:=0$ ). The action of $\mathbb{G}_{a}$ extends to an action of $S L_{2}$, and $V_{k}$ is an irreducible representation of $S L_{2}$.

The supergroup $O S p(1 \mid 2)$ is the group (super)scheme in sVect of automorphisms of the space $\mathbb{k}^{1 \mid 2}$ respecting a fixed symmetric non-degenerate form $\mathbb{k}^{1 \mid 2} \otimes \mathbb{k}^{1 \mid 2} \rightarrow \mathbb{k}_{k}$ and having Berezinian 1 $]^{4}$.

We have: $O S p(1 \mid 2)_{\overline{0}}=S L_{2}$, and

$$
\mathfrak{o s p}(1 \mid 2)=\operatorname{Lie}(O S p(1 \mid 2))
$$

is a (3|2)-dimensional Lie superalgebra with even part $\mathfrak{s l}_{2}$, and the odd part (as a representation of $\mathfrak{s l}_{2}$ ) isomorphic to the standard 2-dimensional representation $V_{1}$. We denote by $h$ the generator of the Cartan subalgebra in $\mathfrak{o s p}(1 \mid 2)_{\overline{1}} \cong \mathfrak{s l}_{2}$ and by $X, Y$ the standard basis of $\mathfrak{o s p}(1 \mid 2)_{\overline{1}} \cong V_{1}$. The elements $h, X, Y$ generate the superalgebra osp $(1 \mid 2)$, with relations

$$
[h, X]=-2 X,[h, Y]=2 Y,[Y, X]=h .
$$

The category $\operatorname{Rep}(O S p(1 \mid 2))$ is semisimple, with isomorphism classes of simple objects (up to parity switch) numbered by even integers: namely, we denote by $\widetilde{M}_{2 k}(k \geq 0)$ the $(k+1 \mid k)$-dimensional irreducible representation such that

$$
\operatorname{Res}_{O S p(1 \mid 2)_{\overline{0}}} \widetilde{M}_{2 k} \cong V_{k+1} \oplus \Pi V_{k}
$$

as $S L_{2}$-representations, and $\mathfrak{o s p}(1 \mid 2)_{\overline{1}}$ acts by odd morphisms accordingly.

\section{The Unipotent ADDitive SUPERGRoup}

3.1. Definition. Let $\mathbb{G}_{a}^{(1 \mid 1)}$ be the (1|1)-dimensional additive algebraic supergroup with $\left(\mathbb{G}_{a}{ }^{(1 \mid 1)}\right)_{\overline{0}}=\mathbb{G}_{a}$. The corresponding (1|1)-dimensional Lie superalgebra has a basis $[x, x], x$, where $x \neq 0$ is an odd element, with relation (the Jacobi identity) $[x,[x, x]]=0$. This defines a Harish-Chandra pair as in [Mas11 and hence an algebraic supergroup.

Denote $\mathcal{U}=\operatorname{Rep}\left(\mathbb{G}_{a}^{(1 \mid 1)}\right)$.

Notation 3.1.1. By $\mathcal{U}_{\text {neat }} \subset \mathcal{U}$ we denote the full subcategory of objects $M \in \mathcal{U}$ such that every indecomposable direct summand of $M$ has non-zero dimension.

3.2. Relation with $O S p(1 \mid 2)$. Let $x \in \mathfrak{o s p}(1 \mid 2)_{\overline{1}}$ be such that $x^{2}=\frac{1}{2}[x, x] \in \mathfrak{o s p}(1 \mid 2)_{\overline{0}}$ corresponds to $\left(\begin{array}{ll}0 & 0 \\ 1 & 0\end{array}\right)$ under the isomorphism $\mathfrak{o s p}(1 \mid 2)_{\overline{0}} \cong \mathfrak{s l}_{2}$. The element $x$ defines an embedding $\mathbb{G}_{a}^{(1 \mid 1)} \hookrightarrow O S p(1 \mid 2)$, which in turn induces a tensor functor

$$
S^{*}: \operatorname{Rep}(O S p(1 \mid 2)) \rightarrow \mathcal{U} .
$$

It is easy to see that $S^{*}\left(\widetilde{M}_{2 k}\right) \cong M_{2 k}$ for any $k \geq 0$.

\footnotetext{
${ }^{4}$ We apologize for assuming connectedness of $O S p(1 \mid 2)$ to avoid the awkward notation $S O S p(1 \mid 2)$
} 
3.3. Clebsh-Gordan coefficients for $\mathbb{G}_{a}^{(1 \mid 1)}$. Let $M_{k}$ be the indecomposable representation of $\mathbb{G}_{a}^{(1 \mid 1)}$ with a basis $a_{0}, a_{1}, \ldots, a_{k}$, with $\overline{a_{j}} \equiv j \bmod 2$ for any $j \geq 0$, and

$$
x . a_{j}= \begin{cases}a_{j+1} & \text { if } j<k \\ 0 & \text { if } j=k\end{cases}
$$

It is easy to see that these are all the indecomposable representations of $\mathbb{G}_{a}^{(1 \mid 1)}$ up to change of parity and isomorphisms.

We have:

$$
\operatorname{Res}_{\mathbb{G}_{a}}^{\mathbb{G}_{a}^{(1 \mid 1)}} M_{2 k+1} \cong V_{k} \oplus \Pi V_{k}, \quad \operatorname{Res}_{\mathbb{G}_{a}}^{\mathbb{G}_{a}^{(1 \mid 1)}} M_{2 k} \cong V_{k} \oplus \Pi V_{k-1}
$$

Example 3.3.1. We have: $M_{0}=\mathbb{1}, \operatorname{Res}_{\mathbb{G}_{a}}^{\mathbb{G}_{a}^{(1 \mid 1)}} M_{1} \cong \mathbb{1} \oplus \Pi \mathbb{1}$.

Lemma 3.3.2. We have

$$
\begin{aligned}
& M_{2 k+1} \simeq \operatorname{Ind}_{\mathbb{G}_{a}^{a}}^{\mathbb{G}_{a}^{(1 \mid 1)}} \Pi V_{k} \\
& \text { or, equivalently, } M_{2 k+1} \simeq U\left(\mathfrak{g}_{a}^{(1 \mid 1)}\right) \otimes_{U\left(\mathfrak{g}_{a}\right)} V_{k} .
\end{aligned}
$$

Remark 3.3.3. Recall that induction between algebraic groups corresponds to coinduction between Lie algebras.

Proof. Immediate straightforward computations.

Lemma 3.3.4. The $\mathbb{G}_{a}^{(1 \mid 1)}$-module decomposition of tensor products of indecomposables into indecomposable summands is as follows:

$$
\begin{aligned}
& M_{2 k} \otimes M_{2 m} \cong \bigoplus_{s=|k-m|}^{k+m} \Pi^{k+m-s} M_{2 s} \\
& M_{2 k+1} \otimes M_{2 m} \cong \bigoplus_{s=m i n(|k-m|,|k-m+1|)}^{k+m} \Pi^{k+m-s} M_{2 s+1} \\
& M_{2 k+1} \otimes M_{2 m+1} \cong \bigoplus_{s=|k-m|}^{k+m} M_{2 s+1} \oplus \Pi M_{2 s+1}
\end{aligned}
$$

Proof. The first identity follows from the Clebsh-Gordan identity for $O S p(1 \mid 2)$ using the fact that $S^{*}\left(\widetilde{M}_{2 k}\right) \cong M_{2 k}$ for any $k \geq 0$. For the second and the third identities we use that for any pair of supergroups $H \subset G$ and $M \in \operatorname{Rep}(H), N \in \operatorname{Rep}(G)$ we have a natural isomorphism

In particular,

$$
\operatorname{Ind}_{H}^{G}(M) \otimes N \cong \operatorname{Ind}_{H}^{G}\left(M \otimes \operatorname{Res}_{H}^{G} N\right) .
$$

$$
M_{2 k+1} \otimes M_{2 m} \cong \operatorname{Ind}_{\mathbb{G}_{a} a}^{\mathbb{G}_{a}^{(1 \mid 1)}} \Pi V_{k} \otimes M_{2 m} \cong \operatorname{Ind}_{\mathbb{G}_{a} a}^{\mathbb{G}_{a}^{(1 \mid 1)}}\left(\Pi V_{k} \otimes\left(V_{m} \oplus \Pi V_{m-1}\right)\right) .
$$

Using the the Clebsh-Gordan coefficients for $S L(2)$ we get

$$
\Pi V_{k} \otimes\left(V_{m} \oplus \Pi V_{m-1}\right)=\bigoplus_{|k-m| \leq s \leq k+m, s \equiv m-k} V_{\bmod 2} \oplus \bigoplus_{|k-m+1| \leq s \leq k+m-1, s \equiv m-k+1} \bmod _{\bmod } \Pi V_{s} .
$$

Hence

$$
\operatorname{Ind}_{\mathbb{G}_{a}}^{\mathbb{G}_{a}^{(1 \mid 1)}}\left(\Pi V_{k} \otimes\left(V_{m} \oplus \Pi V_{m-1}\right)\right)=\bigoplus_{7^{s=m i n}(|k-m|,|k-m+1|)}^{k+m} \Pi^{k+m-s} M_{2 s+1}
$$


The proof of the third identity is similar.

Corollary 3.3.5. The subcategory $\mathcal{U}_{\text {neat }}$ is a Karoubian rigid $S M$ subcategory of $\mathcal{U}$.

Proof. The indecomposable objects in $\mathcal{U}_{\text {neat }}$ are precisely $M_{2 m}$ for $m \geq 0$. By the computation above, the tensor product of any two such $\mathbb{G}_{a}^{1 \mid 1}$-modules lies again in $\mathcal{U}_{\text {neat }}$. So $\mathcal{U}_{\text {neat }}$ is closed under taking tensor products. The remaining claims are straightforward.

3.4. Semisimplification. Consider the semisimplification of $\mathcal{U}$. This is a $\mathbb{k}$-linear monoidal functor $S: \mathcal{U} \rightarrow \overline{\mathcal{U}}$, where $\overline{\mathcal{U}}$ is a semisimple tensor category.

Clearly, $S$ doesn't annihilate any object in $\mathcal{U}_{\text {neat }}$.

Furthermore, we have:

Lemma 3.4.1. The composition

$$
S \circ S^{*}: \operatorname{Rep}(O S p(1 \mid 2)) \longrightarrow \overline{\mathcal{U}}
$$

is a tensor equivalence.

Proof. The composition $S \circ S^{*}$ as in the diagram below

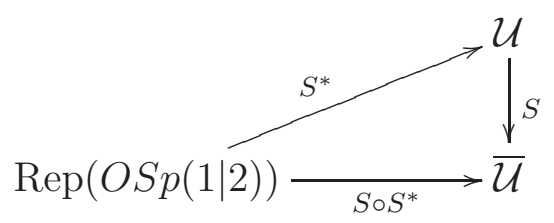

is a $\mathbb{k}$-linear SM functor between semisimple tensor categories. As such, it is automatically exact and faithful, and we only need to check that it is essentially surjective (which will make it automatically full).

Indeed, recall that $\left\{M_{r}\right\}_{r \geq 0}$ are the isomorphism classes of indecomposable objects in $\mathcal{U}$; for odd $r, \operatorname{dim} M_{r}=0$ so $S\left(M_{r}\right)=0$, while for even $r, \operatorname{dim} M_{r} \neq 0$. So

$$
\left\{S\left(M_{2 k}\right)=S \circ S^{*}\left(\widetilde{M}_{2 k}\right)\right\}_{k \geq 0}
$$

are the isomorphism classes of simples in $\overline{\mathcal{U}}$, and $S \circ S^{*}$ is essentially surjective.

3.5. Deligne filtration. Let $x$ be an odd nilpotent element acting on a finite-dimensional superspace $M$. Then $x$ defines a canonical finite increasing filtration 5

$$
\ldots \subset \mathcal{F}^{i}(M) \subset \mathcal{F}^{i+1}(M) \subset \ldots
$$

satisfying the conditions

- $x\left(\mathcal{F}^{i}(M)\right) \subset \mathcal{F}^{i-2}(M)$;

- If $G r^{i}(M):=\mathcal{F}^{i}(M) / \mathcal{F}^{i-1}(M)$ then $x^{i}: G r^{i}(M) \rightarrow \Pi^{i} G r^{-i}(M)$ is an isomorphism for all $i \geq 0$.

In particular, each object in $\mathcal{U}$ is endowed with such a filtration, which is compatible with direct sums. On the indecomposable $\mathbb{G}_{a}^{(1 \mid 1)}$-module $M_{k}$, the filtration is given by:

$$
\mathcal{F}^{k-2 i+1}\left(M_{k}\right)=\mathcal{F}^{k-2 i}\left(M_{k}\right)=\operatorname{span}\left\{a_{j}\right\}_{j \geq i} \text { for } i \geq 0 .
$$

Choose the standard set of generators $h, X, Y$ in $\mathfrak{o s p}(1 \mid 2)$ as in 2.5.

Lemma 3.5.1. For any $\mathbb{G}_{a}^{(1 \mid 1)}$-module $M, G r^{e v}(M):=\bigoplus_{i \in \mathbb{Z}} G r^{2 i}(M)$ has a unique structure of $\mathfrak{o s p}(1 \mid 2)$-module such that $h$ acts by grading and $X$ acts as $G r(x)$.

\footnotetext{
${ }^{5}$ In the case of even $x$ this is the filtration which appears in the Hodge theory.
} 
Proof. In order to define the $\mathfrak{o s p}(1 \mid 2)$-module structure we have to define the action of $Y$ which satisfies the relations. We just write $M$ as a direct sum of modules of the form $M_{2 k}$ and define this structure on each of $M_{2 k}$ in the obvious manner.

Now let us prove uniqueness. Suppose that there are two ways to define $Y, Y^{\prime}$. Then $\left[h, Y-Y^{\prime}\right]=2\left(Y-Y^{\prime}\right)$ and $\left[X, Y-Y^{\prime}\right]=0$. That means that $Y-Y^{\prime} \in \operatorname{End}_{\mathbb{k}}(M)$ is the lowest weight vector of weight 2 with respect to the action of the Lie superalgebra $\mathfrak{o s p}(1 \mid 2)$ generated by $h, X, Y$. Since lowest weight can not be positive, we get $Y-Y^{\prime}=0$.

Let us denote by $T(M)$ the $\mathfrak{o s p}(1 \mid 2)$-module associated to $G r^{e v}(M)$.

Lemma 3.5.2. T defines a $S M$ functor $\operatorname{Rep}\left(\mathbb{G}_{a}^{(1 \mid 1)}\right) \rightarrow \operatorname{Rep}(O S p(1 \mid 2))$ isomorphic to $S$.

Example 3.5.3. We have:

$$
\forall k \in \mathbb{Z}, T\left(M_{2 k+1}\right)=0, T\left(M_{2 k}\right)=\widetilde{M}_{2 k} \text { as vector spaces, }
$$

Proof. First, we check that $T$ is a functor. For this we consider a morphism of $\mathbb{G}_{a}^{(1 \mid 1)}$ modules $\alpha: M \rightarrow N$. It induces the morphism $G r(\alpha): G r^{e v}(M) \rightarrow G r^{e v}(N)$. Note that $\operatorname{Gr}(\alpha)$ commutes with action of $X=G r(x)$ and $h$ and hence with the action of $Y$ by the same argument as in the proof of Lemma 3.5.1. This defines action of $T$ on morphisms and functoriality conditions are straightforward.

Next, we show that $T$ is monoidal.

Consider the filtration on $M \otimes M^{\prime}$, and the subspace $\mathcal{F}^{2 k}(M) \otimes \mathcal{F}^{2 l}\left(M^{\prime}\right)$ for some $k, l$. To determine in which filtration it sits, it is enough to consider this for indecomposable modules $M, M^{\prime}$. Then for any $k, l$ we determine that

$$
\mathcal{F}^{2 k}(M) \otimes \mathcal{F}^{2 l}\left(M^{\prime}\right) \subset \mathcal{F}^{2(k+l)}\left(M \otimes M^{\prime}\right) .
$$

Now, under this embedding, we have:

$$
\mathcal{F}^{2 k-1}(M) \otimes \mathcal{F}^{2 l}\left(M^{\prime}\right)+\mathcal{F}^{2 k}(M) \otimes \mathcal{F}^{2 l-1}\left(M^{\prime}\right) \subset \mathcal{F}^{2(k+l)-1}\left(M \otimes M^{\prime}\right)
$$

Again, it is enough to check this statement for indecomposable modules $M, M^{\prime}$, where this is a direct consequence of the computation of $\mathcal{F}^{i}\left(M_{k}\right)$ given above.

This gives us an embedding $G r^{2 k}(M) \otimes G r^{2 l}\left(M^{\prime}\right) \rightarrow G r^{2 k+2 l}\left(M \otimes M^{\prime}\right)$.

Hence we have a natural transformation

$$
T(M) \otimes T\left(M^{\prime}\right)=\bigoplus_{k, l \in \mathbb{Z}} G r^{2 k}(M) \otimes G r^{2 l}\left(M^{\prime}\right) \rightarrow \bigoplus_{i \in \mathbb{Z}} G r^{2 i}\left(M \otimes M^{\prime}\right)=T\left(M \otimes M^{\prime}\right)
$$

which is an embedding for every $M, M^{\prime}$.

To check that it is a (natural) isomorphism, one again needs to verify this only for indecomposable $M, M^{\prime}$, where it is a direct computation.

We conclude that $T$ is a ( $\mathbb{k}$-linear) monoidal functor.

Clearly, $T$ is essentially surjective (since the essential image of $T$ contains all the simple $\operatorname{OSp}(1 \mid 2)$-modules $\widetilde{M}_{2 k}$ ) and thus full. Thus it is a full monoidal functor into a semisimple category $\operatorname{Rep}(O S p(1 \mid 2))$ and so factors through the functor $S$. The claim now follows.

\section{The Super Jacobson-Morozov Lemma}

\subsection{Definitions.}

Definition 4.1.1. Let $V$ be a vector superspace, and $x \in \operatorname{End}(V)$ an odd nilpotent operator. The element $x$ defines an action of $\mathbb{G}_{a}^{(1 \mid 1)}$ on $V$.

The element $x$ acts neatly in $V$ or if as a $\mathbb{G}_{a}^{(1 \mid 1)}$-module $V$ decomposes into a direct sum of indecomposables $M_{2 k}$ for some $k \in \mathbb{Z}_{\geq 0}$. 
In other words, all indecomposable $\mathbb{G}_{a}^{(1 \mid 1)}$-summands of $V$ have non-zero (super) dimension.

Let $G$ be an algebraic supergroup, and $\mathfrak{g}=\operatorname{Lie}(G)$.

\section{Definition 4.1.2.}

(1) A nilpotent element $x \in \mathfrak{g}_{\overline{1}}$ is called neat if it acts neatly in any finite-dimensional representation of $\mathfrak{g}$.

(2) By $\mathfrak{g}_{\text {neat }}$ we denote the set of all neat nilpotent elements.

Any nilpotent element $x \in \mathfrak{g}_{\overline{1}}$ defines a homomorphism $i_{x}: \mathbb{G}_{a}^{(1 \mid 1)} \rightarrow G$ of algebraic supergroups, and vice versa.

We will call such a homomorphism neat if $x \in \mathfrak{g}_{\text {neat }}$. Clearly, if $x \in \mathfrak{g}_{\text {neat }}$ and $x \neq 0$ then $i_{x}$ is injective.

Let $R: \operatorname{Rep}(G) \longrightarrow \mathcal{U}$ be the restriction functor with respect to the inclusion $i_{x}$. The fact that $i_{x}$ is neat means that $R(M) \in \mathcal{U}_{\text {neat }}$ for any $M \in \operatorname{Rep}(G)$.

Remark 4.1.3. The element $0 \in \mathfrak{g}_{1}$ is always neat.

Example 4.1.4. Let $G=G L(1 \mid 1)$. Then $\mathfrak{g}=\mathfrak{g l}(1 \mid 1)=\operatorname{End}^{\bullet}\left(\mathbb{k}^{1 \mid 1}\right)$ and $\mathfrak{g}_{\overline{1}}$ is spanned by $e:=\left(\begin{array}{ll}0 & 1 \\ 0 & 0\end{array}\right), f:=\left(\begin{array}{ll}0 & 0 \\ 1 & 0\end{array}\right)$.

Then $e, f$ do not act neatly on the faithful $G$-representation $\mathbb{k}^{1 \mid 1}$, so $e, f \notin \mathfrak{g}_{\text {neat }}$. Thus $\mathfrak{g}_{\text {neat }}=\{0\}$ in this case.

Example 4.1.5. Let $G=O S p(1 \mid 2)$. Then $\mathfrak{g}_{\overline{1}}=\mathfrak{g}_{\text {neat }}$.

Example 4.1.6. Let $G=G L(1 \mid 2)$. Then $\mathfrak{g}=\mathfrak{g l}(1 \mid 2)=$ End $^{\bullet}\left(\mathbb{k}^{1 \mid 2}\right)$ and the odd nilpotent cone is

$$
N_{\overline{1}}=\left\{\left(\begin{array}{lll}
0 & a & b \\
c & 0 & 0 \\
d & 0 & 0
\end{array}\right): a c+b d=0\right\}
$$

Now,

$$
\mathfrak{g}_{\text {neat }}=\left\{\left(\begin{array}{ccc}
0 & a & b \\
c & 0 & 0 \\
d & 0 & 0
\end{array}\right) \in N_{\overline{1}}:(a, b) \neq(0,0),(c, d) \neq(0,0)\right\} \cup\{0\} .
$$

This is proved by checking which elements of $\mathfrak{g}_{\overline{1}}$ act neatly on the faithful $G$-representation $\mathbb{k}^{1 \mid 2}$, and using Lemma 6.5.10.

\subsection{Main statement.}

Theorem 4.2.1 (Super Jacobson-Morozov Lemma). Let $G$ be a quasi-reductive algebraic supergroup. Let $i: \mathbb{G}_{a}^{(1 \mid 1)} \hookrightarrow G$ be a neat injective homomorphism. Then the inclusion $i$ can be extended to an injective homomorphism $\bar{i}: O S p(1 \mid 2) \hookrightarrow G$. This extension is unique up to conjugation by an element of $G_{\overline{0}}$.

Proof. Let $R: \operatorname{Rep}(G) \rightarrow \operatorname{Rep}\left(\mathbb{G}_{a}^{(1 \mid 1)}\right)$ be the restriction functor associated with the inclusion $i: \mathbb{G}_{a}^{(1 \mid 1)} \hookrightarrow G$.

Recall that the category $\operatorname{Rep}(G)$ has enough projective objects since $G$ is a quasireductive supergroup (see Proposition 2.3.1).

Now, since we assumed that $i$ is neat, we have: for every projective object $P \in \operatorname{Rep}(G)$, $R(P) \in \mathcal{U}_{\text {neat }}$.

We will show that

$$
\Phi_{i}:=S \circ R: \operatorname{Rep}(G) \rightarrow \overline{\mathcal{U}} \cong \operatorname{Rep}(O S p(1 \mid 2))
$$


is an exact SM $\mathbb{k}$-linear functor, hence inducing a homomorphism $\bar{i}: O S p(1 \mid 2) \hookrightarrow G$. It will clearly be injective (since the supergroup $O S p(1 \mid 2)$ is simple).

Since both $R, S$ are SM and $\mathbb{k}$-linear (hence additive), so is the functor $\Phi_{i}$. So we only need to prove that $\Phi_{i}$ is exact.

First, notice that $\Phi_{i}(P) \neq 0$ for any projective $G$-module $P \neq 0$. Indeed, $R$ is faithful, and $S$ is faithful on the subcategory $\mathcal{U}_{\text {neat }}$ to which $R(P)$ belongs.

Secondly, let

$$
0 \rightarrow M^{\prime} \rightarrow M \rightarrow M^{\prime \prime} \rightarrow 0
$$

be a short exact sequence in $\operatorname{Rep}(G)$. Then for any projective $G$-module $P \neq 0$,

$$
0 \rightarrow P \otimes M^{\prime} \rightarrow P \otimes M \rightarrow P \otimes M^{\prime \prime} \rightarrow 0
$$

is a split exact sequence of projective $G$-modules. Applying $\Phi_{i}$, we obtain a split exact sequence

$$
0 \rightarrow \Phi_{i}(P) \otimes \Phi_{i}\left(M^{\prime}\right) \rightarrow \Phi_{i}(P) \otimes \Phi_{i}(M) \rightarrow \Phi_{i}(P) \otimes \Phi_{i}\left(M^{\prime \prime}\right) \rightarrow 0
$$

in $\overline{\mathcal{U}} \cong \operatorname{Rep}(O S p(1 \mid 2))$.

Now, $\overline{\mathcal{U}} \cong \operatorname{Rep}(O S p(1 \mid 2))$ is a tensor category, so for any $X \in \overline{\mathcal{U}}$, the endofunctor $X \otimes-$ is faithful and exact whenever $X \neq 0$.

Thus $\Phi_{i}(P) \otimes-$ is faithful and exact, so

$$
0 \rightarrow \Phi_{i}\left(M^{\prime}\right) \rightarrow \Phi_{i}(M) \rightarrow \Phi_{i}\left(M^{\prime \prime}\right) \rightarrow 0
$$

is a short exact sequence in $\overline{\mathcal{U}} \cong \operatorname{Rep}(O S p(1 \mid 2))$. This proves that $\Phi_{i}$ is exact.

Since $\Phi_{i}$ is an exact functor, it is isomorphic to the restriction functor $R_{\varphi}$ associated to some homomorphism (embedding) $\varphi: O S p(1 \mid 2) \rightarrow G$.

We fix standard generators $h, X, Y$ in $\mathfrak{o s p}(1 \mid 2)$ as in Section 2.5, and consider the subgroup $\mathbb{G}_{a}^{(1 \mid 1)}$ with the Lie algebra generated by $X$.

Consider the following functors:

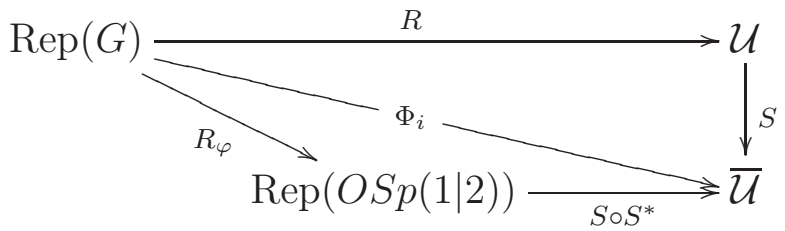

Then we have an isomorphism of functors $\operatorname{Rep}(G) \rightarrow \operatorname{Rep}(O S p(1 \mid 2))$

$$
S \circ S^{*} \circ R_{\varphi} \cong \Phi_{i},
$$

where $S^{*} \circ R_{\varphi}$ is the restriction of $\varphi$ to our chosen $\mathbb{G}_{a}^{(1 \mid 1)} \subset \operatorname{Rep}(O S p(1 \mid 2))$. This makes the diagram of functors above commutative. On every $M \in \operatorname{Rep}(G)$ we have two Deligne filtrations $\mathcal{F}_{i(x)}(M)$ and $\mathcal{F}_{\varphi(X)}(M)$.

Lemma 4.2.2. There exists an automorphism $\psi$ of $\mathfrak{g}$ such that the filtrations $\mathcal{F}_{i(x)}(\mathfrak{g})$ and $\mathcal{F}_{\psi \varphi(X)}(\mathfrak{g})$ coincide.

Proof. Using Lemma 3.5.1 we have $\Phi_{i}(\mathfrak{g})=\operatorname{Gr}_{\mathcal{F}_{i(x)}} \mathfrak{g}$ and $S \circ S^{*} \circ R_{\varphi}(\mathfrak{g})=\operatorname{Gr}_{\mathcal{F}_{\varphi(X)}} \mathfrak{g}$. Both these Lie superalgebras are isomorphic to $\mathfrak{g}$, moreover they are isomorphic to each other as graded Lie superalgebras. This precisely means that the exists an automorphism $\psi$ of $\mathfrak{g}$ such that

$$
\psi \mathcal{F}_{\varphi(X)}(\mathfrak{g})=\mathcal{F}_{\psi \varphi(X)}(\mathfrak{g})=\mathcal{F}_{i(x)}(\mathfrak{g})
$$

Lemma 4.2.3. Let $x_{1}, x_{2} \in \mathfrak{g}$ be two neat elements which define the same Deligne filtration $\mathcal{F}^{\bullet}(\mathfrak{g})$ on $\mathfrak{g}$. Then $x_{1}, x_{2}$ are conjugate with respect to $G_{\overline{0}}$. 
Proof. First, let us note that since $x_{1}, x_{2}$ are neat, both must lie in $\mathcal{F}^{-2}(\mathfrak{g})$ but not in $\mathcal{F}^{-4}(\mathfrak{g})$.

Let $G_{f} \subset G_{\overline{0}}$ be the subgroup preserving the filtration $\mathcal{F}^{\bullet}(\mathfrak{g})$. We have: $\operatorname{Lie}\left(G_{f}\right) \cong$ $\mathcal{F}_{\overline{0}}^{0}(\mathfrak{g})$ (the even part of $\mathcal{F}^{0}(\mathfrak{g})$ ).

Consider the subspace $Q$ of odd elements in $\mathcal{F}^{-2}(\mathfrak{g})$. Then $x_{1}, x_{2} \in Q$. The group $G_{f}$ acts on $Q$. The orbits $O_{1}, O_{2}$ of $x_{1}, x_{2}$ under this action are open, since they have the same tangent space:

$$
T_{x_{i}} O_{i} \cong\left[\operatorname{Lie}\left(G_{f}\right), x_{i}\right]=\mathcal{F}_{\overline{1}}^{-2}(\mathfrak{g})
$$

(the last equality follows from the fact that $x_{1}, x_{2}$ define the same Deligne filtration $\left.\mathcal{F}^{\bullet}(\mathfrak{g})\right)$. Since $O_{1}, O_{2}$ are Zariski open subsets of a vector space, they are dense. Hence they intersect and thus coincide.

Together, Lemmas 4.2.2, 4.2.3 imply that $i(x)=\gamma \varphi(X)$ for some automorphism $\gamma$ of $\mathfrak{g}$. Then $\bar{i}:=\gamma \varphi$ is the desired extension of $i$.

Finally, let us show that $\bar{i}$ is unique up to conjugation in $G_{\overline{0}}$. Indeed, let inclusions $\bar{i}_{1}, \bar{i}_{2}$ : $\mathfrak{o s p}(1 \mid 2) \rightarrow \mathfrak{g}$ coincide on $X \in \mathfrak{o s p}(1 \mid 2)$ and denote by $R_{1}, R_{2}$ corresponding restriction functors $\operatorname{Rep}(G) \rightarrow \operatorname{Rep}(O S p(1 \mid 2))$. We have an isomorphism of functors $S^{*} \circ R_{1} \cong$ $S^{*} \circ R_{2}$ which after composing with $S$ produces an isomorphism $R_{1} \cong R_{2}$. By Tannakian formalism this implies that $\bar{i}_{1}$ and $\bar{i}_{2}$ are conjugate.

The following statement follows from the proof of Theorem 4.2.1.

Corollary 4.2.4. Let $x \in \mathfrak{g}_{\overline{1}}$ be a nilpotent element given by the embedding $i$ in Theorem 4.2.1. Then $x$ is $G_{\overline{0}}$-conjugate to $\varphi(X)$.

Remark 4.2.5. The converse to Theorem 4.2.1 is also true: given a homomorphism $i$ : $\mathbb{G}_{a}^{(1 \mid 1)} \rightarrow G$ which extends to a homomorphism $\bar{i}: O S p(1 \mid 2) \rightarrow G$, the homomorphism $i$ is neat. This follows from the fact that the corresponding restriction functor $R: \operatorname{Rep}(G) \rightarrow$ $\mathcal{U}$ factors through the restriction functor $S^{*}: \operatorname{Rep}(O S p(1 \mid 2)) \rightarrow \mathcal{U}$, so $R(M) \in \mathcal{U}_{\text {neat }}$ for all $M \in \operatorname{Rep}(G)$.

4.3. A corollary. The following corollary of Theorem 4.2 .1 for quasi-reductive supergroups $G$ may be considered as a generalization of the Kostant theorem, which states that there are finitely many nilpotent orbits in the adjoint representation of a semisimple Lie algebra.

Proposition 4.3.1. Let $G$ be a quasi-reductive supergroup. Then there are finitely many $G_{\overline{0}}$-orbits of neat inclusions $i: \mathbb{G}_{a}^{(1 \mid 1)} \hookrightarrow G$. Equivalently, $\mathfrak{g}_{\text {neat }}$ has finitely many $G_{\overline{0}}$-orbits under the adjoint action.

Remark 4.3.2. Equivalently, there exist only finitely many isomorphism classes of tensor functors $R: \operatorname{Rep}(G) \rightarrow \operatorname{Rep}\left(\mathbb{G}_{a}^{(1 \mid 1)}\right)$ whose image lies in $\mathcal{U}_{\text {neat }}$.

Remark 4.3.3. In general, the cone of odd nilpotent elements in $\mathfrak{g}$ might have infinitely many orbits.

Proof. Let $V$ be a faithful finite-dimensional $G$-module (it exists by Remark 2.2.4). Consider the inclusion $\mathfrak{g} \subset \mathfrak{g l}(V)$. There are only finitely many $G L(V)_{\overline{0}}$-orbits in $\mathfrak{g l}(V)_{\text {neat }}$ since there are finitely many non-equivalent representations of $\mathbb{G}_{a}^{(1 \mid 1)}$ in $V$.

Let $O$ be some $G L(V)_{\overline{0}}$-orbit with non-trivial intersection with $\mathfrak{g}_{\overline{1}}$. We will prove that for any $x \in O \cap \mathfrak{g}$ the tangent space $T_{x}\left(G_{\overline{0}} x\right)$ coincides with $T_{x} O \cap \mathfrak{g}$.

Indeed, let $x \in O \cap \mathfrak{g}_{\overline{1}}$. By Lemma 6.5.10, we have: $x \in \mathfrak{g}_{\text {neat }}$. Consider the embedding $x \in \mathfrak{o s p}(1 \mid 2) \subset \mathfrak{g}$. Since $\operatorname{Rep}(\mathfrak{o s p}(1 \mid 2))$ is semisimple we have the osp $(1 \mid 2)$-invariant 
decomposition $\mathfrak{g l}(V)=\mathfrak{g} \oplus W$. That, in particular, implies $\left[x, W_{\overline{0}}\right] \subset W_{\overline{1}}$. Therefore we have

$$
T_{x} O \cap \mathfrak{g}=\left[\mathfrak{g l}(V)_{\overline{0}}, x\right] \cap \mathfrak{g}=\left(\left[\mathfrak{g}_{\overline{0}}, x\right] \oplus\left[W_{\overline{0}}, x\right]\right) \cap \mathfrak{g}=\left[\mathfrak{g}_{\overline{0}}, x\right]=T_{x}\left(G_{\overline{0}} x\right) .
$$

That implies $\operatorname{dim}(O \cap \mathfrak{g})=\operatorname{dim} G_{\overline{0}} x$ for any $x \in O \cap \mathfrak{g}$. Therefore $O \cap \mathfrak{g}$ is a disjoint union of finitely many $G_{\overline{0}}$-orbits. The statement follows.

\subsection{On the set $\mathfrak{g}_{\text {neat }}$.}

Proposition 4.4.1. Let $\mathfrak{g}$ be a Lie superalgebra such that $\mathfrak{g}_{\text {neat }}=\mathfrak{g}_{\overline{1}}$. Then

$$
\mathfrak{g} \cong \mathfrak{g}^{\prime} \oplus \mathfrak{o s p}\left(1 \mid 2 m_{1}\right) \oplus \cdots \oplus \mathfrak{o s p}\left(1 \mid 2 m_{k}\right)
$$

for some $m_{1}, \ldots, m_{k} \in \mathbb{N}$ and a Lie algebra $\mathfrak{g}^{\prime}$.

Proof. We start with the following straightforward observations:

(1) If $\mathfrak{g}_{\text {neat }}=\mathfrak{g}_{\overline{1}}$ then $[x, x] \neq 0$ for any non-zero $x \in \mathfrak{g}_{\overline{1}}$.

(2) If $\mathfrak{g}_{\text {neat }}=\mathfrak{g}_{\overline{1}}$ and $\mathfrak{h}$ is a quotient of $\mathfrak{g}$, then $\mathfrak{h}_{\text {neat }}=\mathfrak{h}_{\overline{1}}$.

We are going to prove the statement by induction on $\operatorname{dim} \mathfrak{g}_{\overline{0}}+\operatorname{dim} \mathfrak{g}_{\overline{1}}$. We note that if $\mathfrak{g}$ is simple then from Kac classification of simple superalgebras (1) holds only for $\mathfrak{g}=\mathfrak{o s p}(1 \mid 2 m)$ or $\mathfrak{g}_{\overline{1}}=0$.

Assume that $\mathfrak{g}_{\text {neat }}=\mathfrak{g}_{\overline{1}}$. Let $\mathfrak{m}$ be some minimal non-zero ideal of $\mathfrak{g}$. Then either $\mathfrak{m}$ is simple or abelian one-dimensional. Note also that by (2) in the latter case $\mathfrak{m}$ is even. If $\mathfrak{m}$ is simple then by above $\mathfrak{m}$ is either a Lie algebra or $\mathfrak{o} \mathfrak{s p}(1 \mid 2 m)$. First assume that $\mathfrak{m} \simeq \mathfrak{o s p}(1 \mid 2 m)$. Then $\mathfrak{g}=\mathfrak{m} \oplus \mathfrak{l}$ with $\operatorname{dim} \mathfrak{l}<\operatorname{dim} \mathfrak{g}$ and the statement follows from the induction assumption.

Now let us assume that $\mathfrak{m}$ is even. Consider $\mathfrak{l}:=\mathfrak{g} / \mathfrak{m}$. Then by the induction assumption

$$
\mathfrak{l} \cong \mathfrak{l}^{\prime} \oplus \mathfrak{o s p}\left(1 \mid 2 m_{1}\right) \oplus \cdots \oplus \mathfrak{o s p}\left(1 \mid 2 m_{k}\right)
$$

for some Lie algebra $\mathfrak{l}^{\prime}$ and $m_{1}, \ldots, m_{k} \in \mathbb{N}$. Set $\mathfrak{s}:=\mathfrak{o s p}\left(1 \mid 2 m_{1}\right) \oplus \cdots \oplus \mathfrak{o s p}\left(1 \mid 2 m_{k}\right)$ and $\mathfrak{n}:=\operatorname{Ker}(\mathfrak{g} \rightarrow \mathfrak{s})$. Consider the exact sequence

$$
0 \rightarrow \mathfrak{n} \rightarrow \mathfrak{g} \rightarrow \mathfrak{s} \rightarrow 0
$$

Since the second cohomology of $\mathfrak{s}$ with coefficients in any module are zero, the above sequence splits. Furthermore, since $\mathfrak{n}$ is purely even the action of $\mathfrak{s}$ on $\mathfrak{n}$ is trivial. Therefore $\mathfrak{g} \cong \mathfrak{n} \oplus \mathfrak{s}$ and the statement follows.

Proposition 4.4.2. Let $G$ be a connected algebraic supergroup with Lie superalgebra $\mathfrak{g}$. Assume that any $x \in \mathfrak{g}_{\overline{1}}$ acts neatly on any representation of $G$. Then

$$
G \cong G^{\prime} \times O S p\left(1 \mid 2 m_{1}\right) \times \cdots \times O S p\left(1 \mid 2 m_{k}\right)
$$

for some $m_{1}, \ldots, m_{k} \in \mathbb{N}$ and an algebraic group $G^{\prime}$.

Proof. The proof is similar to the proof of Proposition 4.4.1. Consider a minimal connected non-trivial normal subgroup $M \subset G$. Since Lie $M$ has no non-zero odd $x$ such that $[x, x]=0$ we get that either $M \simeq O S p(1 \mid 2 m)$ or $M$ is an algebraic group. In the former case we note that the decomposition $\mathfrak{g}=\mathfrak{m} \oplus \mathfrak{l}$ can be lifted to $G=M \times L$ since $O S p(1 \mid 2 m)$ does not have non-trivial discrete normal subgroups. In the latter case, we consider the sequence (11) of Lie superalgebras and by the same reason it induces the decomposition for the groups $G=S \times N$ where $S=O S p\left(1 \mid 2 m_{1}\right) \times \cdots \times O S p\left(1 \mid 2 m_{k}\right)$ and $N$ is an algebraic group.

Corollary 4.4.3. Let $G$ be an algebraic supergroup with Lie superalgebra $\mathfrak{g}$. Assume that any $x \in \mathfrak{g}_{\overline{1}}$ acts neatly on any representation of $G$. Then the (super)dimension of any indecomposable representation of $G$ is not zero. 
Proof. If $G$ is connected we use directly the proposition 4.4.2. Any indecomposable representation of $G$ is of the form $M \otimes L_{1} \otimes \cdots \otimes L_{k}$ where $L_{i}$ is an irreducible representation of $O S p\left(1 \mid 2 m_{i}\right)$ and $M$ is an indecomposable representation of $G^{\prime}$; hence it has non-zero dimension. If $G$ is not connected, denote by $G_{e}$ the connected component of identity. Consider any indecomposable representation $V$ of $G$ and its restriction $\operatorname{Res}_{G_{e}}^{G}(V)$ to $G_{e}$. Decomposing this into a direct sum of indecomposable finite-dimensional $G_{e^{-}}$modules, we see that the finite group $G / G_{e}$ acts on the set of indecomposable direct summands. In other words, there exists an indecomposable $G_{e}$-module $V_{e}$ such that $\operatorname{Res}_{G_{e}}(V)=\oplus_{g} V_{e}^{A d_{g}}$, where $g$ runs over some subset of $G / G_{e}$, and $V_{e}^{A d_{g}}$ denotes the $G_{e}$-module $V_{e}$ with the action twisted by the automorphism $A d_{g}$ of $G_{e}$. Therefore $\operatorname{dim} V$ is a multiple of $\operatorname{dim} V_{e}$ and hence not zero.

Proposition 4.4.4. Let $G$ be quasi-reductive. Assume $\mathfrak{g}_{\text {neat }}=\mathfrak{g}_{\overline{1}}$. Then $\operatorname{Rep}(G)$ is semisimple.

Remark 4.4.5. The condition $\mathfrak{g}_{\text {neat }}=\mathfrak{g}_{\overline{1}}$ means that given a faithful representation $V$ of $G$, any odd element $x \in \mathfrak{g}_{\overline{1}}$ acts neatly. This is also equivalent to the condition that the image of any tensor functor $R: \operatorname{Rep}(G) \rightarrow \operatorname{Rep}\left(\mathbb{G}_{a}^{(1 \mid 1)}\right)$ lies in $\mathcal{U}_{\text {neat }}$.

Proof. Follows from Corollary 4.4.3. Indeed, if $P$ is projective indecomposable, then $\mathbb{k}$ is a direct summand of $P \otimes P^{*}$ which implies that $\mathbb{k}$ is projective.

\section{The minuscule supergroup}

5.1. Let $\mathfrak{m}$ be the Lie superalgebra with basis $\bar{x}, \bar{y}_{n}, \bar{y}_{s}$ such that $\bar{x}$ is even and $\bar{y}_{n}, \bar{y}_{s}$ are odd and

$$
[\bar{x}, \bar{x}]=\bar{y}_{s}+\bar{y}_{n}, \quad\left[\bar{y}_{s}, \bar{y}_{n}\right]=\left[\bar{x}, \bar{y}_{s}\right]=\left[\bar{x}, \bar{y}_{n}\right]=0 .
$$

Consider the category of finite-dimensional $\mathfrak{m}$-modules on which $\bar{y}_{n}$ acts nilpotently and $\bar{y}_{s}$ acts semisimply. By Lemma 5.1 .1 below, this category is equivalent to $\operatorname{Rep}(\mathbb{M})$ for some algebraic pro-supergroup $\mathbb{M}$. We call $\mathbb{M}$ the minuscule supergroup.

Lemma 5.1.1. Let $\mathcal{T}$ be a super Tannakian category which contains $\Pi(\mathbb{1})$ (the unit object with shifted parity). Then $\mathcal{T}$ is equivalent to $\operatorname{Rep}(G)$ for some algebraic pro-supergroup $G$.

Proof. We know from Tannakian formalism that $\mathcal{T}$ is equivalent to $\operatorname{Rep}(\tilde{G}, \varepsilon)$, where $\tilde{G}$ is some supergroup, $\varepsilon: \mu_{2} \rightarrow \tilde{G}$ is a homomorphism and the $g \in \mu_{2}, g \neq 1$ acts by the $\mathbb{Z}_{2}$-grading on objects of $\mathcal{T}$. Let $G$ be the kernel of the representation of $G$ in $\Pi(\mathbb{1})$. Then $\operatorname{Rep}(\tilde{G}, \varepsilon)$ is equivalent to $\operatorname{Rep}(G)$.

Lemma 5.1.2. Let $G$ be an algebraic supergroup, $\mathfrak{g}=\operatorname{Lie}(G)$ and $x \in \mathfrak{g}_{\overline{1}}$. There exists a unique homomorphism $i_{x}: \mathbb{M} \rightarrow G$ such that Lie $\left(i_{x}\right)(\bar{x})=x$.

Proof. Let $y=[x, x] \in \mathfrak{g}_{\overline{0}}$ and $y=y_{s}+y_{n}$ be its Jordan-Chevalley decomposition. Note that $y_{s}, y_{n} \in \mathfrak{g}$ and commute with $x$. Therefore $\mathfrak{h}:=\mathbb{k}\left\langle x, y_{s}, y_{n}\right\rangle$ is a quotient of $\mathfrak{m}$. The homomorphism $\mathfrak{m} \rightarrow \mathfrak{h} \subset \mathfrak{g}$ of Lie superalgebras induces the restriction functor $\operatorname{Rep}(G) \rightarrow \operatorname{Rep}(\mathfrak{m})$. Since $y_{s}$ (resp., $\left.y_{n}\right)$ acts semisimply (resp., nilpotently) on any object of $\operatorname{Rep}(G)$, the above functor defines a faithful SM functor $R_{x}: \operatorname{Rep}(G) \rightarrow \operatorname{Rep}(\mathbb{M})$. The statement follows by Tannakian formalism.

Remark 5.1.3. The above Lemma implies that although Lie( $\mathbb{M})$ is infinite-dimensional, its odd part is one-dimensional. Futhermore, the "even" part $\mathbb{M}_{\overline{0}}$ is a direct product of $\mathbb{G}_{a}$ and the abelian reductive pro-group: the maximal pro-torus of $\mathbb{M}_{\overline{0}}$. 
Remark 5.1.4. The group $\mathbb{M}$ admits the supergroup $\mathbb{G}_{a}^{(0 \mid 1)}$ as the quotient by $\mathbb{M}_{\overline{0}}$, the supergroup $\mathbb{G}_{a}^{(1 \mid 1)}$ as the quotient by the maximal pro-torus of $\mathbb{M}_{\overline{0}}$. Finally, the quotient of $\mathbb{M}$ by $\mathbb{G}_{a} \subset \mathbb{M}_{\overline{0}}$ gives a "superextension" of the maximal pro-torus. Every connected quasireducive supergroup with abelian even part and 1-dimensional odd part is a quotient of this superextension. An example of such a supergroup is $Q(1)$.

Lemma 5.1.5. The semisimplification of $\operatorname{Rep}(\mathbb{M})$ is isomorphic to $\operatorname{Rep}(O S p(1 \mid 2))$.

Proof. We have a fully faithful SM $\mathbb{k}$-linear functor

$$
\mathbf{R}: \mathcal{U} \rightarrow \operatorname{Rep}(\mathbb{M})
$$

corresponding to the quotient map $\mathbb{M} \rightarrow \mathbb{G}_{a}^{(1 \mid 1)}$. Let $S^{\prime}: \operatorname{Rep}(\mathbb{M}) \rightarrow \overline{\operatorname{Rep}(\mathbb{M})}$ denote the semisimplification functor of the category $\operatorname{Rep}(\mathbb{M})$. Then $S^{\prime} \circ \mathbf{R}$ is a full $S M \mathbb{k}$-linear functor from $\mathcal{U}$ to the semisimple SM category $\overline{\operatorname{Rep}(\mathbb{M})}$. Such a functor necessarily factors through the semisimplification

$$
S: \mathcal{U} \rightarrow \operatorname{Rep}(O S p(1 \mid 2))
$$

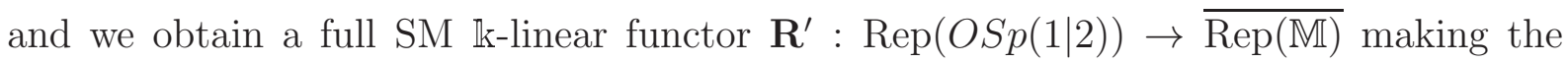
diagram below commutative

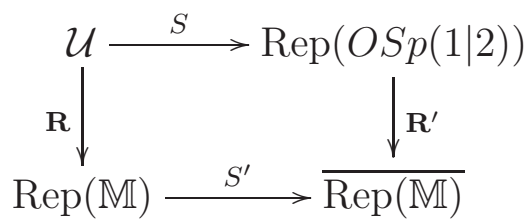

Now, $\operatorname{Rep}(O S p(1 \mid 2))$ is semisimple, so $\mathbf{R}^{\prime}$ is automatically exact and hence faithful. It remains to check that it is essentially surjective, and then we can conclude that it is an equivalence. For this, it is enough to check that any simple object $\bar{M} \in \overline{\operatorname{Rep}(\mathbb{M})}$ lies in the essential image of $\mathbf{R}^{\prime}$.

Indeed, recall that for any such $\bar{M}$ there exists an indecomposable object $M \in \operatorname{Rep}(\mathbb{M})$ such that $\bar{M} \cong S^{\prime}(M)$ and $\operatorname{dim} M \neq 0$.

The semisimple even element $y_{s} \in \operatorname{Lie}(\mathbb{M})$ acts diagonally on $M$; since $M$ is indecomposable, this means that $y_{s}$ acts by $\lambda \operatorname{Id}_{M}$ for some $\lambda \in \mathbb{k}$.

If $\lambda \neq 0$, then the categorical trace (supertrace) of $y_{s}$ on $M$ is

$$
\operatorname{tr}\left(\left.y_{s}\right|_{M}\right)=\operatorname{tr}\left(\left.\left(y_{s}+y_{n}\right)\right|_{M}\right)=\operatorname{tr}\left(\left.[x, x]\right|_{M}\right)=0
$$

(for the leftmost equality, recall that in a tensor category, such as $\operatorname{Rep}(\mathbb{M})$, the trace of a nilpotent endomorphism is necessarily zero). Yet

$$
\operatorname{tr}\left(\left.y_{s}\right|_{M}\right)=\lambda \operatorname{dim}(M)
$$

and we conclude that $\operatorname{dim}(M)=0$ and hence $\bar{M}=0$ (which obviously lies in the essential image of $\mathbf{R}^{\prime}$ ).

If $\lambda=0$, then $y_{s}$ acts trivially on $M$. That is, the action homomorphism $\mathbb{M} \rightarrow G L(M)$ factors through the quotient map $\mathbb{M} \rightarrow \mathbb{G}_{a}^{(1 \mid 1)}$, and hence $M \cong \mathbf{R}(\widetilde{M})$ for some $\widetilde{M} \in \mathcal{U}$. This implies that $\bar{M} \cong S^{\prime} \circ \mathbf{R}(\widetilde{M}) \cong \mathbf{R}^{\prime} \circ S(\widetilde{M})$, and so $\bar{M}$ lies in the essential image of $\mathbf{R}^{\prime}$.

As a corollary of the proof above, we have the following statement: 
Corollary 5.1.6. The full subcategory of $\operatorname{Rep}(\mathbb{M})$ of objects whose indecomposable summands have non-zero dimension is precisely $\mathcal{U}_{\text {neat }}$, embedded in $\operatorname{Rep}(\mathbb{M})$ via the functor $\mathbf{R}$.

\section{General Setting: tensor functors for odd elements}

6.1. Definition. We now consider the most general setting. Let $G$ be an algebraic supergroup with Lie algebra $\mathfrak{g}$. Let $x \in \mathfrak{g}_{\overline{1}}$.

Recall a homomorphism $i_{x}: \mathbb{M} \rightarrow G$ defined in Lemma 5.1.2.

Let $R_{x}: \operatorname{Rep}(G) \rightarrow \operatorname{Rep}(\mathbb{M})$ be the restriction functor with respect to $i_{x}$. Composing $R_{x}$ with the semisimplification functor

$$
S: \operatorname{Rep}(\mathbb{M}) \rightarrow \operatorname{Rep}(O S p(1 \mid 2))
$$

we obtain a SM $\mathbb{k}$-linear functor

$$
\Phi_{x}:=S \circ R_{x}: \operatorname{Rep}(G) \longrightarrow \operatorname{Rep}(O S p(1 \mid 2)) .
$$

Let $\tilde{\mathfrak{g}}:=\Phi_{x}(\mathfrak{g})$. This is an $O S p(1 \mid 2)$-Lie algebra object.

The functor $\Phi_{x}$ is not necessarily exact on either side, but defines a SM $\mathbb{k}$-linear functor

$$
\widetilde{\Phi_{x}}: \operatorname{Rep}(G) \longrightarrow \operatorname{Rep}_{O S p(1 \mid 2)}(\tilde{\mathfrak{g}})
$$

where the latter is the category of $O S p(1 \mid 2)$-equivariant representations of $\tilde{\mathfrak{g}}$.

Remark 6.1.1. It is not hard to see that if $\mathfrak{g}$ is one of the classical superalgebras $\mathfrak{g l}(V), \mathfrak{o} \mathfrak{s p}(V), \mathfrak{q}(V)$ or $\mathfrak{p}(V)$ then $\tilde{\mathfrak{g}}$ is a classical superalgebra of the same type. Also in all examples we know if $\mathfrak{g}$ is quasi-reductive then $\tilde{\mathfrak{g}}$ is quasi-reductive but we do not know if it is true in general.

6.2. Special case: nilpotent odd operator. If $x$ is nilpotent, then $[x, x]$ is a nilpotent even element, and so the embedding $\mathbb{M} \hookrightarrow G$ factors through the homomorphism $\mathbb{M} \rightarrow$ $\mathbb{G}_{a}^{(1 \mid 1)}$. In that case, we will have a restriction functor $R: \operatorname{Rep}(G) \rightarrow \operatorname{Rep}\left(\mathbb{G}_{a}^{(1 \mid 1)}\right)$ as in Section 4. Composing with the semisimplification functor $S: \operatorname{Rep}\left(\mathbb{G}_{a}^{(1 \mid 1)}\right) \rightarrow \operatorname{Rep}(O S p(1 \mid 2))$, we obtain a $\mathbb{k}$-linear SM functor

$$
\Phi_{x}:=S \circ R: \operatorname{Rep}(G) \longrightarrow \operatorname{Rep}(O S p(1 \mid 2)) .
$$

When $x$ is neat, this is precisely the functor considered in Theorem 4.2.1, the restriction functor with respect to some embedding $O S p(1 \mid 2) \rightarrow G$.

Remark 6.2.1. Let $x_{1}, x_{2} \in \mathfrak{g}_{\text {neat }}$. By Tannakian formalism, $\Phi_{x_{1}} \cong \Phi_{x_{2}}$ iff $x_{1}, x_{2}$ are conjugate with respect to $G_{\overline{0}}$.

6.3. Back to general case. Let $x \in \mathfrak{g}_{\overline{1}}$ and $[x, x]=y_{s}+y_{n}$. Then $x$ acts nilpotently on $M^{y_{s}}$. Consider the Deligne filtration

$$
\ldots \subset \mathcal{F}^{i}\left(M^{y_{s}}\right) \subset \mathcal{F}^{i+1}\left(M^{y_{s}}\right) \subset \ldots
$$

as defined in Section 3.5.

Lemma 6.3.1. $\Phi_{x}(M) \cong T\left(M^{y_{s}}\right)$ where $T=G r^{e v}$ as defined in Lemma 3.5.2.

Proof. Note that $y_{s}$ acts semisimply on $M$ and any eigenspace of $y_{s}$ in $\mathbb{M}$-invariant. Every indecomposable $\mathbb{M}$-submodule which lies in the eigenspace with non-zero eigenvalue has superdimension zero as explained in the proof of Lemma 5.1.5. Hence the statement follows from Lemma 3.5.2. 
6.4. Relation to the DS functor. We now describe a special case of the construction above.

Let $\mathbb{G}_{a}^{(0 \mid 1)}$ be the purely odd affine additive group, with Lie $\left(\mathbb{G}_{a}^{(0 \mid 1)}\right)=\mathbb{k}^{0 \mid 1}$ (a purely odd vector superspace with trivial bracket).

A representation of $\mathbb{G}_{a}^{(0 \mid 1)}$ is defined by a pair $(x, M)$ where $x: M \rightarrow \Pi M$ is an odd endormophism of $M$, such that $[x, x]=0$.

Consider the group homomorphism $\mathbb{G}_{a}^{(1 \mid 1)} \rightarrow \mathbb{G}_{a}^{(0 \mid 1)}$ and the corresponding (faithful, exact, $\mathbb{k}$-linear SM) embedding

$$
I: \operatorname{Rep}\left(\mathbb{G}_{a}^{(0 \mid 1)}\right) \longrightarrow \mathcal{U}=\operatorname{Rep}\left(\mathbb{G}_{a}^{(1 \mid 1)}\right) .
$$

For any $M \in \operatorname{Rep}\left(\mathbb{G}_{a}^{(0 \mid 1)}\right)$, the indecomposable summands of $I(M)$ in $\mathcal{U}$ then have (categorical) dimensions either \pm 1 (the torsion part of $M$ seen as a module over the algebra of dual numbers $k[x] / x^{2}$ ) or 0 (free part of $M$ over $k[x] / x^{2}$ ). These first summands are not annihilated by the semisimplification functor $S: \operatorname{Rep}\left(\mathbb{G}_{a}^{(1 \mid 1)}\right) \rightarrow \operatorname{Rep}(O S p(1 \mid 2))$, and are sent to representations with trivial $O S p(1 \mid 2)$-action; the summands of second type are annihilated by the semisimplification functor $S$. Hence

$$
S \circ I: \operatorname{Rep}\left(\mathbb{G}_{a}^{(0 \mid 1)}\right) \longrightarrow \operatorname{Rep}(O S p(1 \mid 2))
$$

is in fact given by a $\mathbb{k}$-linear SM functor

$$
D: \operatorname{Rep}\left(\mathbb{G}_{a}^{(0 \mid 1)}\right) \longrightarrow \text { sVect }
$$

which sends $M$ to its homology $\operatorname{Ker}\left(\left.x\right|_{M}\right) / x M$.

Given an embedding $\mathbb{G}_{a}^{(0 \mid 1)} \hookrightarrow G$ into an algebraic supergroup $G$, consider $\mathfrak{g}:=\operatorname{Lie}(G)$ as a Lie algebra object in $\operatorname{Rep}(G)$, and let $\tilde{\mathfrak{g}}:=D(\mathfrak{g})$. The functor $D$ induces a $\mathbb{k}$-linear SM functor

$$
\operatorname{Rep}(G) \longrightarrow \operatorname{Rep}(\tilde{\mathfrak{g}})
$$

which is precisely the Duflo-Serganova functor. Hence $\widetilde{\Phi_{x}}$ is a generalization of the DufloSerganova functor.

Remark 6.4.1. In general the functor $\Phi_{x}$ does not satisfy the Hinich property ("exact in the middle"), satisfied by the Duflo-Serganova functors (see [DuS05]). For example, for $G=\mathbb{G}_{a}^{(1 \mid 1)}$ and $x \in \operatorname{Lie}(G)_{\overline{1}} \backslash\{0\}$, the functor $\Phi_{x}$ is just the semisimplification functor $S$. Consider the short exact sequence of $\mathbb{G}_{a}^{(1 \mid 1)}$-modules:

$$
0 \rightarrow M_{1} \rightarrow M_{2} \rightarrow M_{0} \rightarrow 0
$$

Then $S\left(M_{1}\right)=0, S\left(M_{2}\right)=\widetilde{M}_{2}, S\left(M_{0}\right)=\widetilde{M}_{0}$. This implies that the complex

$$
0 \rightarrow S\left(M_{1}\right) \rightarrow S\left(M_{2}\right) \rightarrow S\left(M_{0}\right) \rightarrow 0
$$

is not exact at the middle.

6.5. Support of a module. Let $G$ be an algebraic supergroup, and $\mathfrak{g}=\operatorname{Lie}(G)$.

Definition 6.5.1. Let $M \in \operatorname{Rep}(G)$. We define the support of $M$, denoted $\operatorname{supp}(M)$, to be the set of all such $x \in \mathfrak{g}_{\overline{1}}$ that satisfy: $\Phi_{x}(M) \neq 0$.

Remark 6.5.2. Note that by definition $\operatorname{supp}(0)=\emptyset$ and $0 \in \operatorname{supp}(M)$ for all non-zero $M \in \operatorname{Rep}(G)$.

Lemma 6.5.3. Let $M, N \in \operatorname{Rep}(G)$.

(1) $\operatorname{supp}(M \oplus N)=\operatorname{supp}(M) \cup \operatorname{supp}(N)$.

(2) $\operatorname{supp}(M \otimes N)=\operatorname{supp}(M) \cap \operatorname{supp}(N)$. 
Proof. The statements follow from the fact that the functor $\Phi_{x}$ is additive and monoidal.

Remark 6.5.4. The support of a module is not necessarily open nor closed. This can be seen from Proposition 6.5.8 and the Example 4.1.6, which shows that for $G=G L(1 \mid 2)$ the support of any projective module is $\mathfrak{g}_{\text {neat }}$ and it is neither open nor closed.

Below we give some results about the relation between neat elements, support, and projective modules.

\section{Proposition 6.5.5.}

$$
\bigcap_{M \in \operatorname{Rep}(G), M \neq 0} \operatorname{supp}(M)=\mathfrak{g}_{\text {neat }}
$$

Proof. First, let us show that for any $M \in \operatorname{Rep}(G), \mathfrak{g}_{\text {neat }} \subset \operatorname{supp}(M)$. Indeed, let $x \in \mathfrak{g}_{\text {neat }}$, let $i_{x}: \mathbb{G}_{a}^{(1 \mid 1)} \rightarrow G$ be the corresponding homomorphism and $R_{x}: \operatorname{Rep}(G) \rightarrow \mathcal{U}$ the corresponding restriction.

If $M \neq 0$, then $R_{x}(M)$ has at least one indecomposable summand, and since $x$ is neat, this summand is of non-zero dimension. Hence $\Phi_{x}(M) \neq 0$, and $x \in \operatorname{supp}(M)$. This proves

$$
\mathfrak{g}_{\text {neat }} \subset \bigcap_{M \in \operatorname{Rep}(G), M \neq 0} \operatorname{supp}(M) .
$$

Next, we prove the inclusion in the other direction.

Let $x \in \mathfrak{g}_{1}$. Let $i_{x}: \mathbb{M} \rightarrow G$ be the corresponding homomorphism and $R_{x}: \operatorname{Rep}(G) \rightarrow$ $\operatorname{Rep}(\mathbb{M})$ the corresponding restriction.

Assume that $x \in \operatorname{supp}(M)$ for all non-zero $M \in \operatorname{Rep}(G)$; that is, $\Phi_{x}(M) \neq 0$ for all non-zero $M \in \operatorname{Rep}(G)$.

By Lemma 6.5.6 below, this means that for all $M \in \operatorname{Rep}(G)$, the indecomposable summands of $R_{x}(M)$ have non-zero dimension. By Corollary 5.1.6, this implies that

$$
R_{x}(M) \in \mathcal{U}_{\text {neat }} \subset \mathcal{U} \subset \operatorname{Rep}(\mathbb{M}) \text { for all } M \in \operatorname{Rep}(G) .
$$

The fact that $R_{x}(M) \in \mathcal{U}$ for all $M$ implies that the embedding $i_{x}$ factors through the quotient map $\mathbb{M} \rightarrow \mathbb{G}_{a}^{(1 \mid 1)}$, and so $x$ is nilpotent. The fact that $R_{x}(M) \in \mathcal{U}_{\text {neat }}$ for all $M$ implies that $x \in \mathfrak{g}_{\text {neat }}$, as required.

Lemma 6.5.6. Let $x \in \mathfrak{g}_{\overline{1}}$ and $M \in \operatorname{Rep}(G)$ be such that $R_{x}(M)$ has at least one indecomposable summand of dimension 0 (so $x$ is not "neat" on $M$ ). Then the functor

$$
\Phi_{x}: \operatorname{Rep}(G) \rightarrow \operatorname{Rep}(O S p(1 \mid 2))
$$

annihilates some non-zero module in $\operatorname{Rep}(G)$.

Proof. Consider the vector superspaces $M$ and $\Phi_{x}(M)$. The latter can be identified with a subspace of $R_{x}(M)$ which is the direct sum of all summands of $R_{x}(M)$ which have non-zero superdimension.

Since $\Phi_{x}$ is a SM functor, for every partition $\lambda$ we have:

$$
S^{\lambda}(M)=0 \text { implies } S^{\lambda}\left(\Phi_{x}(M)\right)=0 .
$$

As vector spaces, the dimension of $M$ is greater than that of $\Phi_{x}(M)$. Hence these vector superspaces are not isomorphic. This implies (cf. De02, EnHS15) that there exists a partition $\lambda$ such that $S^{\lambda}(M) \neq 0$ while $S^{\lambda}\left(\Phi_{x}(M)\right)=0$. Hence $\Phi_{x}\left(S^{\lambda}(M)\right)=0$ while $S^{\lambda}(M) \neq 0$, as required. 
The following lemma is useful for determining the support of projective modules (Proposition 6.5.8).

Lemma 6.5.7. Let $\mathcal{T}, \mathcal{T}^{\prime}$ be two tensor categories, and $F: \mathcal{T} \rightarrow \mathcal{T}^{\prime}$ be a $S M \mathbb{k}$-linear functor (not necessarily exact). Assume that $F$ annihilates some object $M \neq 0$. Then $F$ annihilates all projective objects in $\mathcal{T}$.

Proof of 6.5.7. The map ev : $M \otimes M^{*} \rightarrow \mathbb{1}$ is surjective in any tensor category, and $F(e v)=0$. Let $P$ be a projective object in $\mathcal{T}$. Then $\operatorname{Id}_{P} \otimes e v: P \otimes M \otimes M^{*} \rightarrow P$ is surjective so it splits. Since $F\left(\operatorname{Id}_{P} \otimes e v\right)=0$, we conclude that $F(P)=0$.

Proposition 6.5.8. For any non-zero projective module $P$ in $\operatorname{Rep}(G)$, we have: $\operatorname{supp}(P)=\mathfrak{g}_{\text {neat }}$.

Proof. By Proposition 6.5.5, we have:

$$
\mathfrak{g}_{\text {neat }} \subset \operatorname{supp}(P) .
$$

In the other direction, let $x \in \mathfrak{g}_{\overline{1}}$, and assume $x \notin \mathfrak{g}_{\text {neat }}$. By Proposition 6.5.5, there exists $M \in \operatorname{Rep}(G)$ such that $\Phi_{x}(M)=0$. Thus by Lemma 6.5.7, $\Phi_{x}(P)=0$ for all projective $P$, so $x \notin \operatorname{supp}(P)$.

We conjecture that for quasi-reductive supergroups the converse of the statement in Proposition 6.5.8 also holds (cf. [DuS05]):

Conjecture 1. Let $G$ be a quasi-reductive group, and $M \in \operatorname{Rep}(G)$. If $\operatorname{supp}(M)=\mathfrak{g}_{\text {neat }}$, then $M$ is projective.

Remark 6.5.9. The intersection of $\operatorname{supp}(M)$ with the self-commuting cone

$$
\mathcal{N}_{\text {comm }}:=\left\{x \in \mathfrak{g}_{\overline{1}}:[x, x]=0\right\}
$$

has been studied extensively in [DuS05] (there it is called the associated variety of $M$ ).

In particular, it has been shown there that for quasi-reductive supergroups $G$ of KacMoody type, we have:

$$
M \in \operatorname{Rep}(G) \text { is projective iff } \operatorname{supp}(M) \cap \mathcal{N}_{\text {comm }}=\{0\} .
$$

In such cases, we have: $\mathfrak{g}_{\text {neat }} \cap \mathcal{N}_{\text {comm }}=\{0\}$ by Proposition 6.5.8, and this implies Conjecture 1. However, it would be interesting to obtain this result in greater generality.

Finally, in the lemma below, we give a convenient criterion to determine when $x \in \mathfrak{g}_{\text {neat }}$.

Lemma 6.5.10. Let $G$ be a quasi-reductive algebraic supergroup, and $V$ be a faithful representation of $G$. Let $x \in \mathfrak{g}_{\overline{1}}$, and assume $\left.x\right|_{V}$ is nilpotent and neat.

Then $x$ is nilpotent and $x \in \mathfrak{g}_{\text {neat }}$.

Proof. Let $R_{x}: \operatorname{Rep}(G) \rightarrow \operatorname{Rep}(\mathbb{M})$ be the restriction functor associated to $x$.

Let $\mathcal{A} \subset \operatorname{Rep}(G)$ be the full subcategory of (finite) direct sums of $G$-modules of the form $V^{\otimes r} \otimes\left(V^{*}\right)^{\otimes s}, r, s \in \mathbb{Z}_{\geq 0}$. Let $\operatorname{Kar}(\mathcal{A})$ be the full subcategory of $\operatorname{Rep}(G)$ whose objects are direct summands of objects in $\mathcal{A}$. Since $V$ is faithful, any projective module sits in $\operatorname{Kar}(\mathcal{A})$.

The full subcategory $\mathcal{U}_{\text {neat }} \subset \operatorname{Rep}(\mathbb{M})$ is closed under taking direct sums, direct summands and tensor products.

Since $\left.x\right|_{V}$ is nilpotent and neat, we have: $R_{x}(V) \in \mathcal{U}_{\text {neat }}$, so $R_{x}(M) \in \mathcal{U}_{\text {neat }}$ for all $M \in \operatorname{Kar}(\mathcal{A})$ (in particular, this implies that the group homomorphism $i_{x}: \mathbb{M} \rightarrow G$ factors through the homomorphism $\mathbb{M} \rightarrow \mathbb{G}_{a}^{(1 \mid 1)}$ and so $x$ is nilpotent).

Thus $\Phi_{x}(P) \neq 0$ for any projective module $P \in \operatorname{Rep}(G), P \neq 0$. Since we assumed that $G$ is quasi-reductive, there exists at least one projective module $P \neq 0$ in $\operatorname{Rep}(G)$. Using Proposition 6.5.8, we conclude that $x \in \operatorname{supp}(P)=\mathfrak{g}_{\text {neat }}$. 


\section{Reductive EnVELOPES}

7.1. Let $\mathcal{C}_{\text {neat }} \subset \operatorname{Rep}(G)$ be the full subcategory with objects $M$ so that any $x \in \mathfrak{g}_{\overline{1}}$ is neat on $M$. This is a full Karoubi additive rigid SM subcategory.

Let $S: \mathcal{C}_{\text {neat }} \rightarrow \overline{\mathcal{C}_{\text {neat }}}$ be the semisimplification of $\mathcal{C}_{\text {neat }}$.

Proposition 7.1.1. Let $G$ be an algebraic supergroup. Then $\overline{\mathcal{C}_{\text {neat }}} \cong \operatorname{Rep}(\bar{G})$ for some reductiv 6 algebraic pro-supergroup $\bar{G}$. If any indecomposable object in $\mathcal{C}_{\text {neat }}$ has non-zero dimension then we have a homomorphism $G \rightarrow \bar{G}$.

\section{Example 7.1.2.}

(1) For a purely even supergroup $G=G_{\overline{0}}$ the group $\bar{G}$ is the reductive envelope $G_{\overline{0}}^{\text {red }}$ of the algebraic group $G_{\overline{0}}$, AK02].

(2) For $G=\mathbb{G}_{a}^{(1 \mid 1)}$ or $G=\mathbb{M}$, we have: $\mathcal{C}_{\text {neat }}=\mathcal{U}_{\text {neat }}$, and we obtain $\bar{G} \cong O S p(1 \mid 2)$. Note that for $G=\mathbb{M}$, the homomorphism $G \rightarrow \bar{G}$ is not injective.

Proof. First of all, notice that the category $\overline{\mathcal{C}_{\text {neat }}}$ is super-Tannakian. Indeed, by Deligne's theorem (see [De02]), every object in $\operatorname{Rep}(G)$ is annihilated by some Schur functor, and so the same holds for every object in $\mathcal{C}_{\text {neat }}$. The semisimplification functor $\mathcal{C}_{\text {neat }} \rightarrow \overline{\mathcal{C}_{\text {neat }}}$ is $\mathrm{SM}$, so every object in $\overline{\mathcal{C}_{\text {neat }}}$ is annihilated by some Schur functor. Applying Deligne's theorem again, we conclude that $\overline{\mathcal{C}_{\text {neat }}}$ is super-Tannakian. Since $\Pi(\mathbb{1})$ is an object of $\overline{\mathcal{C}_{\text {neat }}}$, Lemma 5.1.1 implies $\overline{\mathcal{C}_{\text {neat }}} \cong \operatorname{Rep}(\bar{G})$ for some algebraic pro-supergroup $\bar{G}$. Furthermore, the category $\overline{\mathcal{C}_{\text {neat }}}$ is semisimple. Hence $\bar{G}$ is reductive.

It remains to check that there is a section $S^{*}: \overline{\mathcal{C}_{\text {neat }}} \rightarrow \mathcal{C}_{\text {neat }}$, which will induce a homomorphism $G \rightarrow \bar{G}$. But this is a consequence of [EtO18, Corollary 3.11].

Proposition 7.1.3. If $G$ is quasi-reductive then any indecomposable $M$ in $\mathcal{C}_{\text {neat }}$ has nonzero dimension.

Proof. Take any indecomposable representation $V \in \mathcal{C}_{\text {neat }}$.

Let $K=\operatorname{Ker}(G \rightarrow G L(V))$, and let $G^{\prime}=G / K$. The supergroup $G^{\prime}$ is also quasireductive: its even part $G_{\overline{0}}^{\prime}$ is a quotient of the reductive group $G_{\overline{0}}$, so it is also reductive.

Now, $V$ has a natural structure of an indecomposable faithful representation of $G^{\prime}$ on which any $x \in \operatorname{Lie}\left(G^{\prime}\right)_{\overline{1}}$ is neat. By Lemma 6.5.10, we obtain: $\operatorname{Lie}\left(G^{\prime}\right)_{\overline{1}}=\operatorname{Lie}\left(G^{\prime}\right)_{\text {neat }}$.

Hence by Proposition 4.4.4, the category $\operatorname{Rep}\left(G^{\prime}\right)$ is semisimple.

This implies that $\operatorname{dim} V \neq 0$, since any non-zero indecomposable object in a semisimple tensor category has non-zero dimension.

Remark 7.1.4. If we drop the assumption that $G$ is quasi-reductive then it is not true in general that the dimension of any indecomposable object in $\mathcal{C}_{\text {neat }}$ is not 0 . For example, consider the supergroup $G=\mathbb{G}_{a} \times \mathbb{G}_{a}^{(1 \mid 1)}$ and $V=M_{2} \oplus \Pi M_{2}$ as a module over $\mathfrak{g}_{a}^{(1 \mid 1)}$. Let $\left\{v_{1}, v_{2}, v_{3}\right\}$ and $\left\{w_{1}, w_{2}, w_{3}\right\}$ be bases of $M_{2}$ and $\Pi M_{2}$ respectively with action of odd generator $X$ given by $X\left(v_{i}\right)=v_{i+1}$ and $X\left(w_{i}\right)=w_{i+1}$ for $i=1,2$ and $X\left(v_{3}\right)=X\left(w_{3}\right)=0$. Define the action of a generator $y \in \operatorname{Lie}\left(\mathbb{G}_{a}\right)$ on $V$ by

$$
y\left(v_{1}\right)=w_{2}, y\left(v_{2}\right)=w_{3}, y\left(v_{3}\right)=y\left(w_{1}\right)=y\left(w_{2}\right)=y\left(w_{3}\right)=0 .
$$

Then $V$ is an indecomposable $G$-module of superdimension 0 .

Remark 7.1.5. Let $G$ be an algebraic supergroup, and let $\widetilde{G}$ be the fundamental (super)group of the super-Tannakian category $\operatorname{Rep}(G)$. The algebraic supergroup $\widetilde{G}$ is isomorphic to the semidirect product $\mu_{2} \rtimes G$. If $\widetilde{G} \cong \mu_{2} \times G$ then there is a homomorphism

\footnotetext{
${ }^{6}$ An algebraic supergroup $G$ is called reductive if $\operatorname{Rep}(G)$ is semisimple.
} 
$\varepsilon: \mu_{2} \rightarrow G$ and we have a decomposition of abelian categories

$$
\operatorname{Rep}(G) \cong \operatorname{Rep}(G, \varepsilon) \oplus \Pi \operatorname{Rep}(G, \varepsilon) .
$$

Consider the full Karoubi additive subcategory $\mathcal{C}_{\text {neat }}^{\prime}:=\mathcal{C}_{\text {neat }} \cap \operatorname{Rep}(G, \varepsilon)$ of $\mathcal{C}_{\text {neat }}$. Taking its semisimplification $\overline{\mathcal{C}_{\text {neat }}^{\prime}}$, the same argument as in the proof of Proposition 7.1 .1 shows that $\overline{\mathcal{C}_{\text {neat }}^{\prime}} \cong \operatorname{Rep}(\bar{G}, \bar{\varepsilon})$ for a reductive algebraic supergroup $\bar{G}$ and $\bar{\varepsilon}: \mu_{2} \rightarrow \bar{G}$. If the assumption of Proposition 7.1.1 holds then there exists a homomorphism $\phi: G \rightarrow \bar{G}$ such that $\phi \circ \varepsilon=\bar{\varepsilon}$.

In particular, for $G=G_{\overline{0}}$ purely even and $\varepsilon$ the trivial morphism, $\bar{G} \cong G_{\overline{0}}^{\text {red }}$ and $\overline{\mathcal{C}_{\text {neat }}^{\prime}}$ is equivalent to $\operatorname{Rep}_{\text {vect }}\left(G_{\overline{0}}^{\text {red }}\right)$.

If $G=\mathbb{G}_{a}^{(1 \mid 1)}$ or $\mathbb{M}$, then $\widetilde{G}$ does not split into direct product of $G$ and $\mu_{2}$ and hence the abelian tensor category $\operatorname{Rep}(G)$ does not have splitting. However, the Karoubian category $\mathcal{C}_{\text {neat }}$ has a splitting

$$
\mathcal{C}_{\text {neat }}=\mathcal{C}_{\text {neat }}^{\prime} \oplus \Pi \mathcal{C}_{\text {neat }}^{\prime},
$$

where $\mathcal{C}_{\text {neat }}^{\prime}$ is the Karoubian tensor subcategory generated by $\Pi M_{2}$. Then $\overline{\mathcal{C}_{\text {neat }}^{\prime}}$ is equivalent to $\operatorname{Rep}(O S p(1 \mid 2), \varepsilon)$ where $\varepsilon$ is the isomorphism of $\mu_{2}$ with the center of $O S p(1 \mid 2)_{\overline{0}}$.

\section{REFERENCES}

[AK02] Y. Andr, B. Kahn, and P. OSullivan, Nilpotence, radicaux et structures monodales, Rendiconti del Seminario Matematico della Universit di Padova 108 (2002): 107-291; arXiv:0203273 (2002).

[BEEO20] J. Brundan, I. Entova-Aizenbud, P. Etingof, V. Ostrik, Semisimplification of the category of tilting modules for $G L_{n}$, arXiv:2002.01900 (2020).

[De02] P. Deligne, Catégories tensorielles, Mosc. Math. J. 2 (2002), no. 2, 227-248; https://www.math.ias.edu/files/deligne/Tensorielles.pdf.

[DuS05] M. Duflo, V. Serganova, On associated variety for Lie superalgebras, arXiv:math/0507198 (2005).

[EnHS15] I. Entova-Aizenbud, V. Serganova, V. Hinich, Deligne categories and the limit of categories $\operatorname{Rep}(G L(m \mid n))$, IMRN, rny144 (2018); arXiv:1511.07699 (2015).

[EtGNO15] P. Etingof, S. Gelaki, D. Niksych, V. Ostrik, Tensor Categories, Math. Surveys Monogr., Volume 205, AMS (2015).

[EtO18] P. Etingof, V. Ostrik, On semisimplification of tensor categories, arXiv:1801.04409 (2018).

[H15] Th. Heidersdorf, On supergroups and their semisimplified representation categories, Algebras and Representation Theory 22, no. 4, p. 937-959 (2019); arXiv:1512.03420.

[Mas11] A. Masuoka, Harish-Chandra pairs for algebraic affine supergroup schemes over an arbitrary field. Transformation Groups 17.4 (2012): 1085-1121; arXiv:1111.2387 (2011).

[Mil17] J. Milne, Algebraic Groups: The Theory of Group Schemes of Finite Type over a Field, (Cambridge Studies in Advanced Mathematics). Cambridge: Cambridge University Press (2017).

Inna Entova-Aizenbud, Dept. of Mathematics, Ben Gurion University, Beer-Sheva, ISRAEL.

E-mail address: entova@bgu.ac.il

Vera Serganova, Dept. of Mathematics, University of California at Berkeley, BerkeLEY, CA 94720.

E-mail address: serganov@math.berkeley.edu 